\title{
Expression levels and associations of five long non-coding RNAs in gastric cancer and their clinical significance
}

\author{
HELEN BARONG BINANG ${ }^{1,2}$, YUN-SHAN WANG ${ }^{1}$, MARLVIN ANEMEY TEWARA $^{3}$, LUTAO DU ${ }^{1}$, \\ SHUANG SHI ${ }^{1}$, NING LI ${ }^{1}$, ARISTON GABRIEL ABAKUNDANA NSENGA ${ }^{1,2}$ and CHUANXIN WANG ${ }^{1}$ \\ ${ }^{1}$ Department of Clinical Laboratory, The Second Hospital of Shandong University, Jinan, Shandong 250033; \\ ${ }^{2}$ Department of Clinical Laboratory Diagnostics, School of Medicine, Shandong University; ${ }^{3}$ Department of Biostatistics, \\ School of Public Health, Cheeloo College of Medicine, Shandong University, Jinan, Shandong 250012, P.R. China
}

Received July 13, 2019; Accepted December 12, 2019

DOI: $10.3892 / 01.2020 .11311$

\begin{abstract}
Gastric cancer (GC) is a type of cancer that is commonly diagnosed worldwide due to a lack of early diagnostic, prognostic and therapeutic targets for this disease. The aim of the present study was to examine the expression levels of five long non-coding RNAs, namely PTPRG antisense RNA 1 (PTPRG-AS1), forkhead box P4 antisense RNA 1 (FOXP4-AS1), bladder cancer-associated transcript 2 (BLACAT2), ZXF2 and upregulated in colorectal cancer (UCC), to study their associations with patient characteristics and assess their prognostic efficacy, in order to determine the possibility of their application as GC biomarkers. The expression levels of long non-coding RNAs (lncRNAs) were determined by reverse transcription-quantitative PCR analysis of 61 pairs of GC tissues and adjacent healthy gastric mucosa tissues and GC cell lines. The Chi-square test was conducted to assess the associations of lncRNA expression levels with clinical characteristics of patients. The effect of UCC on GC cell proliferation was determined using in vitro functional experiments. The prognostic efficacy of FOXP4-AS1, BLACAT2 and UCC were examined in the Gene Expression Profiling Interactive Analysis database and those of PTPRG-AS1 were examined in the Kaplan Meier Plot database. Gene alteration frequencies of PTPRG-AS1 and BLACAT2 in GC were identified using the
\end{abstract}

Correspondence to: Professor Chuanxin Wang, Department of Clinical Laboratory, The Second Hospital of Shandong University, 247 Beiyuan Road, Jinan, Shandong 250033, P.R. China

E-mail: cxwang@sdu.edu.cn

Abbreviations: ANTs, adjacent normal tissues; BLACAT2, bladder cancer-associated transcript 2; CCK-8, cell counting kit-8; DFS, disease free survival; GC, gastric cancer; HER2, human epidermal growth factor receptor 2; lncRNA, long non-coding RNA; LNM, lymph node metastasis; OS, overall survival; RT-qPCR, reverse transcription-quantitative PCR; siRNA, small interfering RNA; UCC, upregulated in colorectal cancer

Key words: cell proliferation, disease-free survival, gastric cancer, long non-coding RNAs, overall survival, relative expression
cBioPortal for Cancer Genomics. PTPRG-AS1, FOXP4-AS1, BLACAT2, ZXF2 and UCC were found to be upregulated in GC cell lines and GC tissues compared with adjacent normal tissues. PTPRG-AS1 and ZXF2 expression levels were associated with the expression status of the cell proliferation marker Ki67. UCC promoted the proliferation of GC cells in vitro and was associated with lymph node metastasis. Increased expression of FOXP4-AS1 indicated a favorable outcome in terms of disease-free survival, whereas high expression of PTPRG-AS1 was associated with poor survival rates for patients in different GC risk groups. BLACAT2 gene mutation was associated with poor disease-free survival outcome for patients with GC. The results suggest that PTPRG-AS1, FOXP4-AS1, BLACAT2, ZXF2 and UCC are potential biomarkers for the detection of $\mathrm{GC}$ at the molecular level and may be used as potential targets for GC therapy. The individual roles of these lncRNAs may be utilized for prognostic predictions.

\section{Introduction}

Gastric cancer (GC), or stomach adenocarcinoma, was the most common malignancy of the gastrointestinal system, according to statistics in 2017 (1). It was the second leading cause of cancer-associated mortalities worldwide in 2018 and is a regularly diagnosed form of cancer (2). The GC cases in China in 2017 represented approximately $42 \%$ of all cases worldwide (3). This may be due to the highly documented incidence of Helicobacter pylori infection among the Chinese population $(1,3)$. Throughout the years, several attempts have been made to identify methods for ameliorating GC-associated mortality. Efforts have been directed towards the discovery of sensitive diagnostic biomarkers and specific prognostic markers and therapeutic targets. Nevertheless, minimal progress has been made, as GC remains a major global health burden with a median survival of only 3-5 months (4). Thus, enhanced comprehension of the molecular and genetic changes that accompany gastric tumorigenesis is essential. Also important is the identification of oncogenes that promote gastric tumor growth. These oncogenes may serve as biomarkers for GC progression and as molecular therapeutic targets.

Genomic studies have revealed that approximately $90 \%$ of the human genome is actively transcribed (5). Only $2 \%$ is 
transcribed into protein-coding genes, whereas the remainder is transcribed into non-protein-coding genes (6). Non-coding RNAs consist of microRNAs (miRNAs/miRs), small interfering RNAs (siRNAs) and long non-coding RNAs (lncRNAs). lncRNAs are RNA transcripts over 200 nucleotides in length. Their expression is highly cell- and tissue-specific (7). They affect various processes of protein, DNA and RNA interactions, thereby regulating their expression and functions. Consequently, lncRNAs are capable of potently altering the proliferative, invasive and metastatic potential of malignant cells (8). They have been implicated in various types of cancer, such as colorectal, non-small cell lung, non-muscle invasive bladder, breast and prostate cancers (9-13), although their functional mechanisms are largely underexplored. Recently, certain studies have focused on the analyses of lncRNA expression patterns and their associations with clinical and demographic characteristics of patients. Of note, several lncRNAs have been associated with tumor growth, metastasis and patient clinical outcomes $(1,14,15)$.

lncRNAs drive several oncogenic processes $(16,17)$. Findings have demonstrated that the expression of lncRNAs is dysregulated between tumor and adjacent normal tissues $(18,19)$, and between cancer patients and healthy subjects $(20,21)$. IncRNAs are increasingly gaining attention as potential biomarkers and therapeutic targets for various kinds of malignancies (22). Specific GC biomarkers are currently limited. Although advancements have been made and a number of treatment options are available, the survival rates of patients with GC have not been markedly improved. For instance, trastuzumab, a human epidermal growth factor receptor 2 (HER2) antibody, has been recommended as the first-line regimen for advanced $\mathrm{GC}$ treatment (16). However, only HER2 ${ }^{+}$patients, which correspond to $12-20 \%$ of GC cases, may benefit from this regimen (22). Further understanding of the molecular mechanisms and exploration of corresponding GC biomarkers could contribute to the early detection and optimal prediction of prognosis. Identification of GC-associated lncRNAs could lead to the development of novel markers and effective therapeutic targets for patients with GC.

The lncRNA PTPRG antisense RNA 1 (PTPRG-AS1) is the antisense of protein tyrosine phosphatase receptor type G (PTPRG), a tumor suppressor gene. High expression of PTPRG-AS1 has been linked to poor clinical outcomes for lung cancer patients (23). In breast cancer, high expression of this lncRNA has been suggested to downregulate the expression of PTPRG (24), thereby enhancing disease progression.

Forkhead box P4 antisense RNA 1 (FOXP4-AS1) is an lncRNA associated with colorectal cancer (25). It is significantly upregulated in colorectal cancer tissues, promotes cell proliferation and inhibits apoptosis both in vitro and in vivo (25).

The lncRNA LINC00958, also termed bladder cancer-associated transcript 2 (BLACAT2), has been found to be markedly upregulated in lymph node-metastatic bladder cancer and was associated with lymph node metastasis (LNM) (26). It is implicated in bladder cancer-associated lymphangiogenesis and lymphatic metastasis. Patients with bladder cancer and high BLACAT2 expression have been shown to have shorter overall and metastasis-free survival (26).

lncRNA ZXF2 is highly expressed in lung adenocarcinoma (27). It is believed that ZXF2 enhances lung cancer progression via the c-myc/e-cadherin pathway. Suppression of
ZXF2 inhibits c-myc, a potent proto-oncogene, and enhances the expression of e-cadherin, a tumor suppressor gene. In addition, ZXF2 is located adjacent to c-myc in chromosome locus $8 \mathrm{q} 24.2$, which is a hotspot for multiple types of human cancer. Interaction of ZXF2 and c-myc results in cell cycle progression, proliferation, migration and invasion of tumor cells. Upregulation of ZXF2 was also associated with lymph node metastasis and poor prognosis (27).

Huang et al (15) demonstrated that the long intergenic non-coding RNA ENST00000602992 (CTD-2227E11.1), an oncogenic lncRNA also known as upregulated in colorectal cancer (UCC), promotes the progression of colorectal cancer by upregulating KRAS and acting as a sponge for miR-143. Furthermore, it was shown that suppression of UCC in colorectal cancer cells inhibited proliferation and invasion, and induced apoptosis. A positive association was also observed between UCC expression, LNM and overall survival (OS) of patients with colorectal cancer.

Thus far, limited data exist on the expression profiles, functions and prognostic efficacy of PTPRG-AS1, FOXP4-AS1, BLACAT2, ZXF2 and UCC in GC. The present study aimed to examine their expression profiles in GC tissues and cell lines and to determine their functions and clinical significance in GC. It was found that lncRNAs PTPRG-AS1, ZXF2, FOXP4-AS1, BLACAT2 and UCC are upregulated in GC tissues and cell lines. Also reported in the present study are the functions, clinical significance and potential for application of the aforementioned lncRNAs as diagnostic, prognostic and therapeutic markers in GC.

\section{Materials and methods}

Study design. Based on previous studies, nine lncRNAs aberrantly expressed in other adenocarcinomas, namely UCC, FOXP4-AS1, SCAL1, ANRASSF1, PTPRG-AS1, ZXF2, SChLAP1, BLACAT2 and SBF2-AS1 (5,13,15,23,25-29), were selected as potential candidates in the present study. This selection was based on the fact that many types of cancer, though substantially different, share common gene expression signatures. In the screening stage, the expression levels of the nine lncRNAs were examined in GC tissues and five upregulated lncRNAs became the focus of subsequent studies.

Patients and specimens. The current study was approved through the Clinical Research Ethics Committee of the Second Hospital of Shandong University. Written informed consent was obtained from all participants. In total, 61 pairs of GC tissues and adjacent normal tissues (ANTs) collected at the Second Hospital of Shandong University were used in the present study. All patients were confirmed to have GC via histopathological evaluation. None of the included patients had received pre-operative chemotherapy or radiotherapy. The obtained samples were immediately snap-frozen in liquid nitrogen and then stored at $-80^{\circ} \mathrm{C}$ until further use for total RNA extraction.

Cell culture. The non-tumorigenic human gastric epithelial cell line GES-1 and four GC cell lines, namely MKN-45 (indicated not to have a TP53 mutation), NCI-N87, AGS and HGC-27, which were purchased from the American Type Culture 
Collection and maintained in liquid nitrogen or at $-80^{\circ} \mathrm{C}$ in the laboratory, were used in the present study. All the cells were cultured in RPMI-1640 medium (Invitrogen; Thermo Fisher Scientific, Inc.). All culture media were supplemented with 10\% FBS (Gibco; Thermo Fisher Scientific, Inc.), 100 U/ml penicillin and $100 \mu \mathrm{g} / \mathrm{ml}$ streptomycin. The cells were plated and incubated in $5 \% \mathrm{CO}_{2}$ at $37^{\circ} \mathrm{C}$ and $95 \%$ humidity.

Total RNA extraction and reverse transcription-quantitative $P C R(R T-q P C R)$. Total RNA was extracted from the tissue samples using TRIzol reagent (Thermo Fisher Scientific, Inc.) and from the cell lines using the miRNeasy Mini Kit (Qiagen N.V.), according to the manufacturers' protocol. The quantity and quality of extracted RNA were determined by a NanoDrop spectrophotometer (Thermo Fisher Scientific, Inc.). For RT-qPCR, $1 \mu \mathrm{g}$ of RNA was reverse transcribed to cDNA using a Takara reverse transcription kit (Takara Biotechnology Co., Ltd.). RT-qPCR analyses were conducted on a CFX-96 real-time PCR System (Bio-Rad Laboratories, Inc.) using SYBR Premix Ex TaqTM (Takara Biotechnology Co., Ltd.). The temperature protocol was as follows: $37^{\circ} \mathrm{C}$ for $15 \mathrm{~min}$, followed by $85^{\circ} \mathrm{C}$ for $5 \mathrm{sec}$. The thermocycling conditions were as follows: $95^{\circ} \mathrm{C}$ for $30 \mathrm{sec}$, followed by 40 cycles of $95^{\circ} \mathrm{C}$ for $5 \mathrm{sec}, 60^{\circ} \mathrm{C}$ for $30 \mathrm{sec}$ and $72^{\circ} \mathrm{C}$ for $30 \mathrm{sec}$, followed by a final step of $72^{\circ} \mathrm{C}$ for $2 \mathrm{~min}$. The primer sequences used for target amplification are listed in Table SI. Data were normalized to GAPDH expression. Relative expression levels were determined using the comparative $2^{-\Delta \Delta \mathrm{Cq}}$ method (30).

UCC siRNA transfection. Three siRNA sequences specifically targeted against UCC and si-NC, a scramble siRNA used as negative control, were synthesized from Shanghai GenePharma Co., Ltd. The AGS cells were transfected with the siRNA oligonucleotides at a concentration of $20 \mu \mathrm{M}$ using Lipofectamine ${ }^{\circledR} 2000$ reagent (Invitrogen; Thermo Fisher Scientific, Inc.), according to the manufacturers' protocol. The nucleotide sequences of the siRNAs are listed in Table SI. The cells were harvested for further study $24 \mathrm{~h}$ after transfection. The efficiency of siRNA-mediated knockdown was determined using RT-qPCR by comparing the expression of UCC in siRNA-treated cells with that in si-NC-treated cells.

Cell proliferation assay. To determine the effect of UCC on cell proliferation, a cell proliferation assay was performed using Cell Counting kit-8 (CCK8) according to the manufacturer's protocol. AGS cells transfected with si-UCC were seeded into 96 -well plates at a density of $3 \times 10^{3}$ cells/well and incubated for 24,48 and $72 \mathrm{~h}$, respectively. si-NC treatment was used as the negative control. The effect of UCC on cell proliferation was determined using CCK-8 (Wuhan Boster Biological Technology, Ltd.) as follows: At the end of each 24, 48 or $72 \mathrm{~h}$ incubation, $10 \mu \mathrm{l}$ of CCK-8 solution was added to each well. After $4 \mathrm{~h}$, the number of viable cells was determined by measuring the absorbance of water-soluble formazan at $465 \mathrm{~nm}$ using a FlexStation 3 plate reader (Molecular Devices, LLC). The experiments were performed in six replicates.

Colony formation assay. Transfected AGS cells were seeded in 6 -well plates at a density of $1 \times 10^{3} /$ well and incubated at $37^{\circ} \mathrm{C}$ and $5 \% \mathrm{CO}_{2}$ for 14 days. Subsequently, colonies were stained with $10 \%$ Giemsa, visualized under a light microscope (Olympus Corporation) at x100 magnification and then physically quantified. The experiments were performed in duplicate.

Survivalanalysis.SurvivalanalysesforFOXP4-AS1,BLACAT2 and UCC were conducted using Gene Expression Profiling Interactive Analysis (GEPIA; http://gepia.cancer-pku.cn), an online tool (31). A dataset from an independent cohort (Affymetrix ID: 232242_at) was utilized for the evaluation of PTPRG-AS1 expression as a prognostic factor of GC. The results from the survival analyses of $631 \mathrm{GC}$ tissue samples from The Cancer Genome Atlas (http://cancer.gov/tcga) are available at the Kaplan Meier Plot database (http://kmplot. com) (32-34). Gene alteration frequencies of PTPRG-AS1 and BLACAT2 mRNA in GC were assessed using cBioPortal for Cancer Genomics (http://www.cbioportal.org) (35).

Statistical analysis. Statistical analyses were performed using SPSS software (version 22.0; IBM Corp.) and GraphPad Prism (GraphPad Software, Inc.). Data were presented as the mean \pm standard deviation. Differences between experimental groups were assessed by the Student's t-test. Associations between lncRNA expression and clinical characteristics were analyzed using the simple Chi-square test. $\mathrm{P}<0.05$ was considered to indicate a statistically significant difference.

\section{Results}

PTPRG-AS1, FOXP4-AS1, BLACAT2, ZXF2 and UCC are upregulated in $G C$ tissues and cell lines. To determine the relative expression of the lncRNAs in GC, their expression levels were examined in GC cell lines and GC tissues in comparison with ANTs. Fig. 1 shows the relative expression and mean-fold changes of PTPRG-AS1 ( $\mathrm{P}=0.001$; Fig. 1A), FOXP4-AS1 $(\mathrm{P}<0.001$; Fig. 1B), BLACAT2 ( $\mathrm{P}<0.001$; Fig. 1C), ZXF2 $(\mathrm{P}=0.102$, Fig. 1D) and UCC $(\mathrm{P}=0.012$; Fig. $1 \mathrm{E})$ in 61 pairs of GC tissues and ANTs. All five lncRNAs were upregulated in GC tissues compared with their corresponding ANTs. Apart from ZXF2, relative expression levels of the lncRNAs in GC tissues were significant compared with those observed in ANTs. The expression levels of the five lncRNAs were verified in four GC cell lines, specifically MKN-45, NCI-N87, AGS and HGC-27, relative to the non-tumorigenic human gastric epithelial cell line, GES-1. All five lncRNAs were highly expressed in GC cell lines. Elevated PTPRG-AS1 expression was observed in two cell lines, MKN-45 and NCI-N87. FOXP4-AS1 was highly expressed in three cell lines, AGS, NCI-N87 and MKN-45. BLACAT2 and ZXF2 were upregulated in all four cell lines and UCC was upregulated in AGS, NCI-N87 and HGC-27 compared with the GES-1 cell line (Fig. 2A-E). These results demonstrate that PTPRG-AS1, FOXP4-AS1, BLACAT2, ZXF2 and UCC are upregulated in GC tissues and cell lines.

PTPRG-AS1, FOXP4-AS1, BLACAT2, ZXF2 and UCC are associated with the clinical characteristics of patients with $G C$. In order to study the roles of these lncRNAs in GC, the associations between overexpression and the clinical characteristics of patients were evaluated. Table I summarizes the associations between IncRNA expression levels and 
A
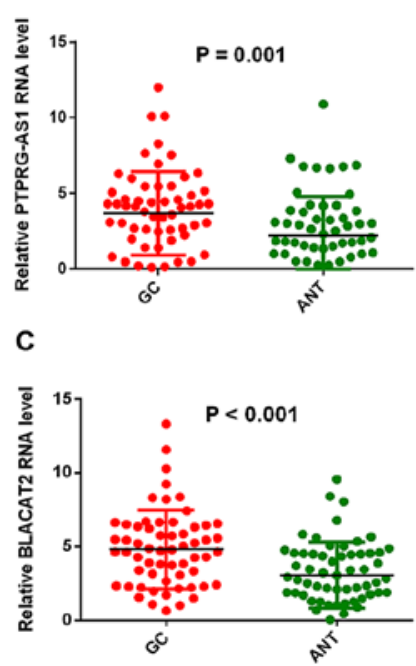

E

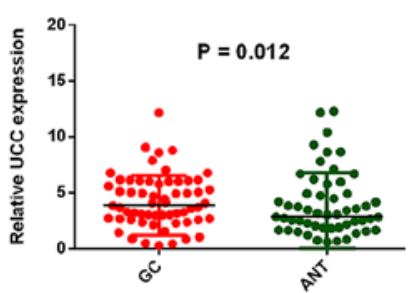

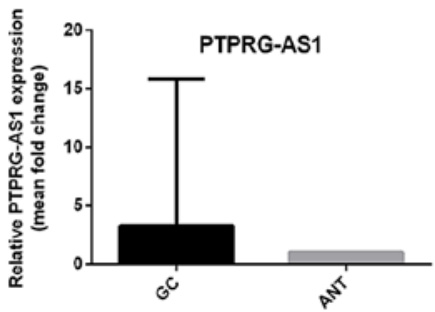
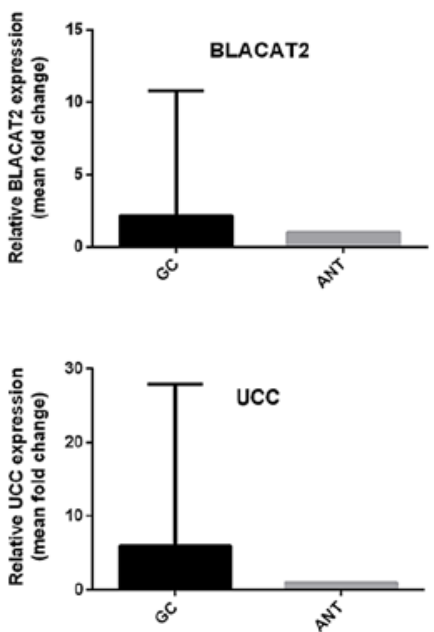

B

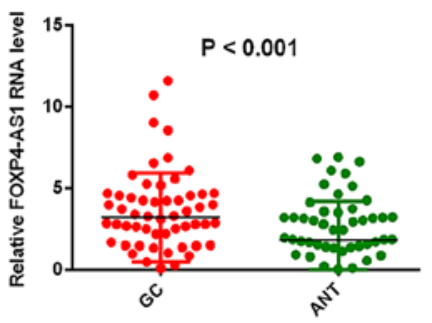

D
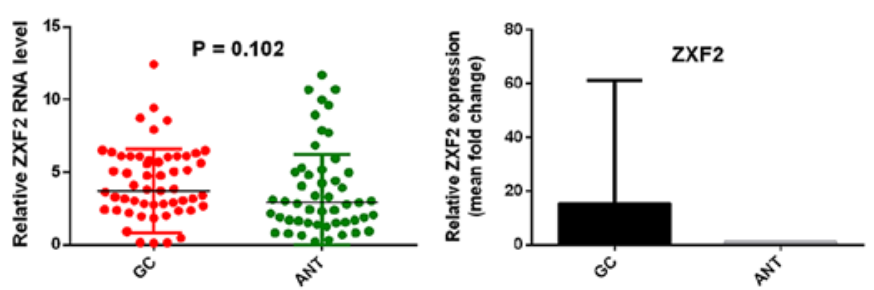

Figure 1. PTPRG-AS1, FOXP4-AS1, BLACAT2, ZXF2 and UCC were upregulated in GC tissues. Reverse transcription-quantitative PCR results demonstrated that the levels of (A) PTPRG-AS1, (B) FOXP4-AS1, (C) BLACAT2, (D) ZXF2, and (E) UCC in 61 pairs of GC tissues were higher than those in ANTs. Error bars represent the mean \pm SD. GC, gastric cancer, ANT, adjacent normal tissues; PTPRG-AS1, PTPRG antisense RNA 1; FOXP4-AS1, forkhead box P4 antisense RNA 1; BLACAT2, bladder cancer-associated transcript 2; UCC, upregulated in colorectal cancer.

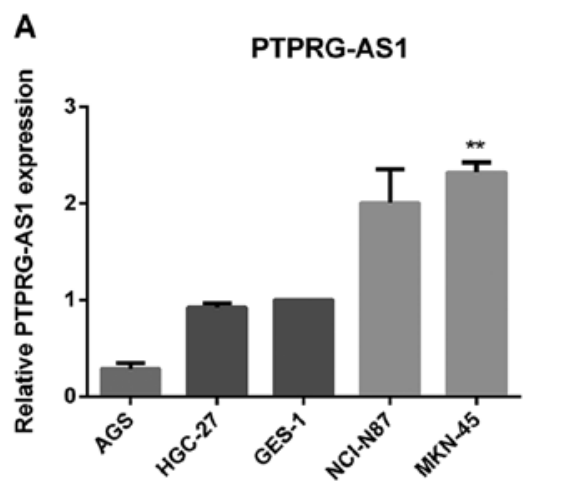

B
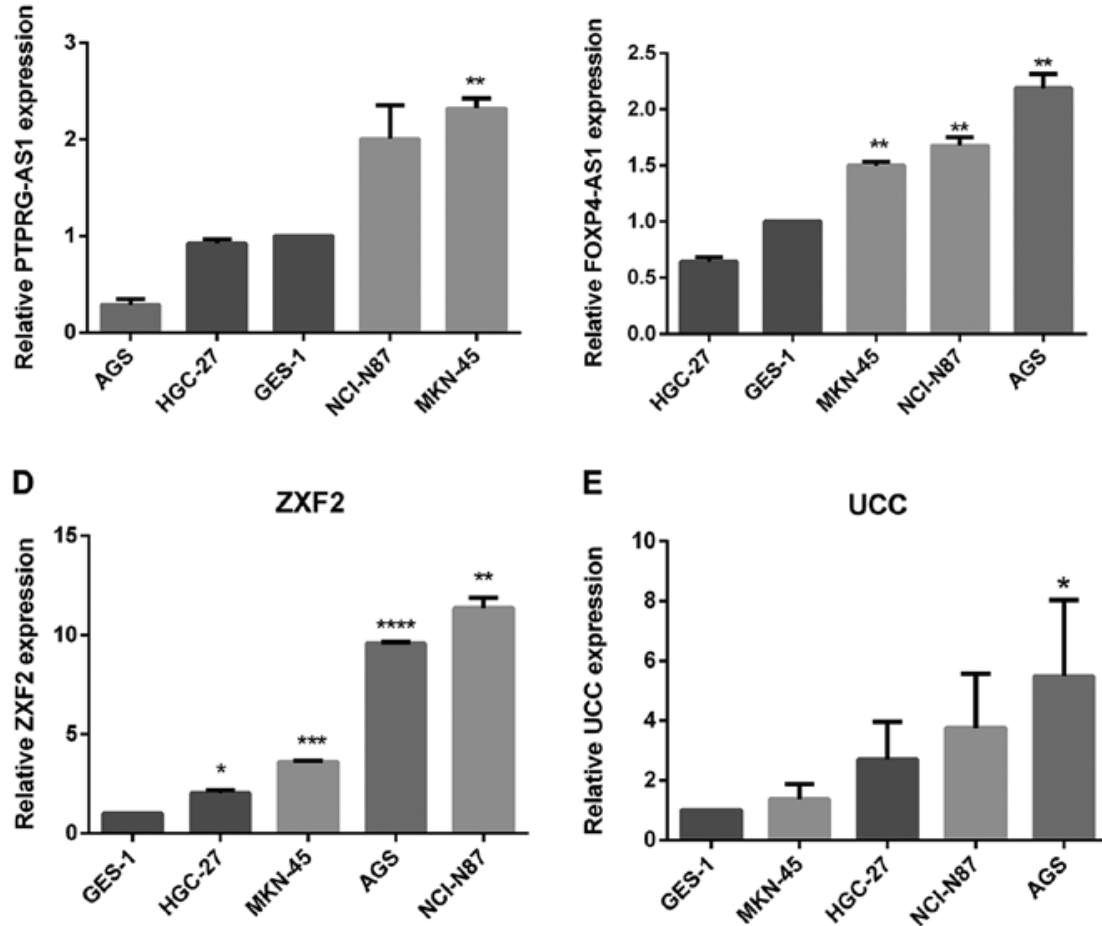

E

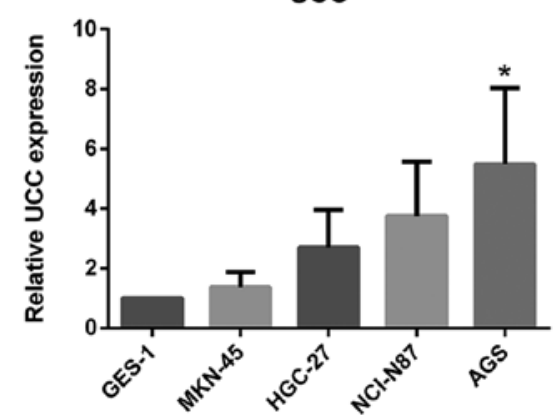

C

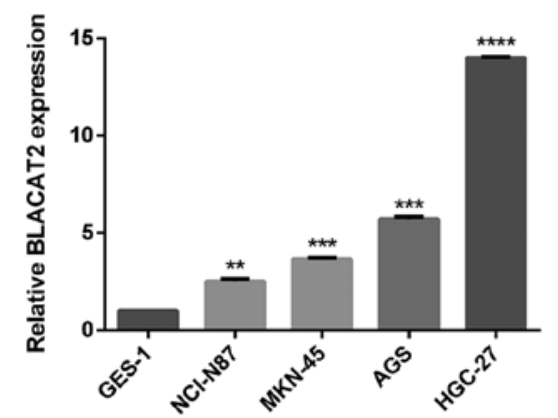

Figure 2. Expression of lncRNAs in GC cell lines. Reverse transcription-quantitative PCR results revealed the baseline mRNA levels of (A) PTPRG-AS1, (B) FOXP4-AS1, (C) BLACAT2, (D) ZXF2, and (E) UCC in the non-tumorigenic gastric epithelial cell line GES-1 and four gastric cancer cell lines. The expression levels of IncRNAs were normalized to that of GES-1. Error bars represent the mean \pm SD of triplicate experiments. ${ }^{*} \mathrm{P}<0.05,{ }^{* * *} \mathrm{P}<0.01,{ }^{* * * *} \mathrm{P}<0.001$ and ${ }^{* * * * *} \mathrm{P}<0.0001$. IncRNAs, long non-coding RNAs; PTPRG-AS1, PTPRG antisense RNA 1; FOXP4-AS1, forkhead box P4 antisense RNA 1; BLACAT2, bladder cancer-associated transcript 2; UCC, upregulated in colorectal cancer. 


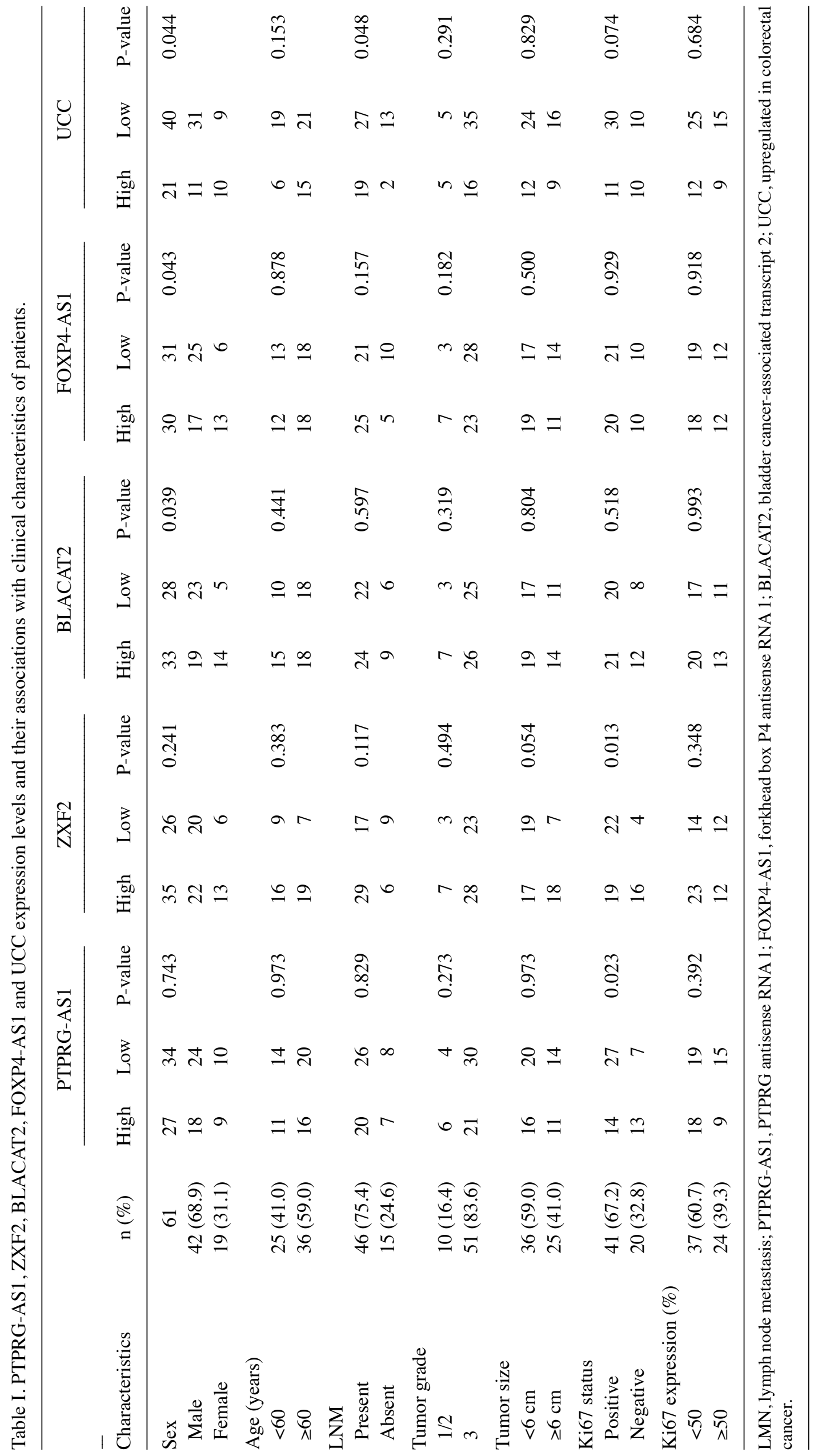


A
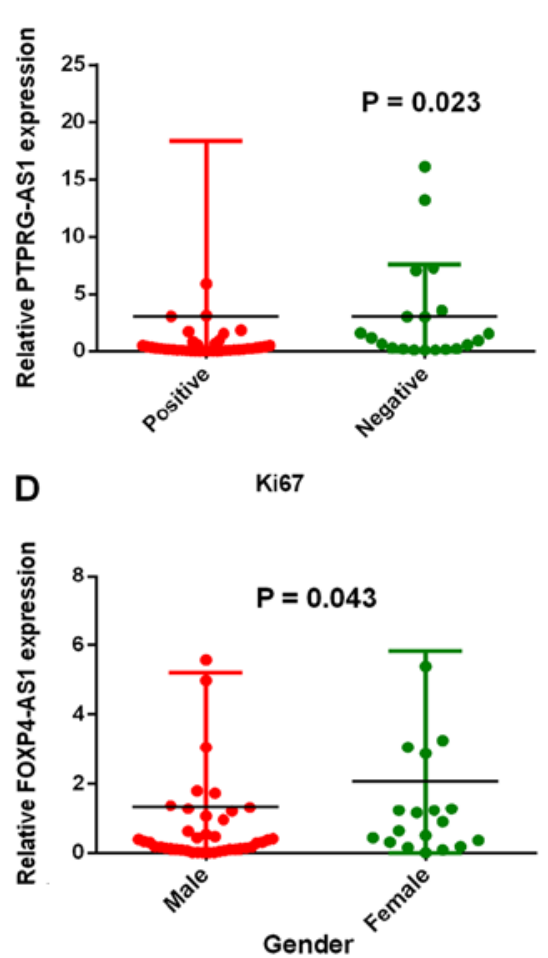

G

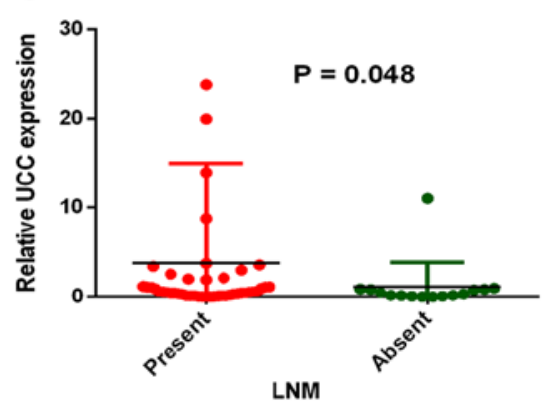

B

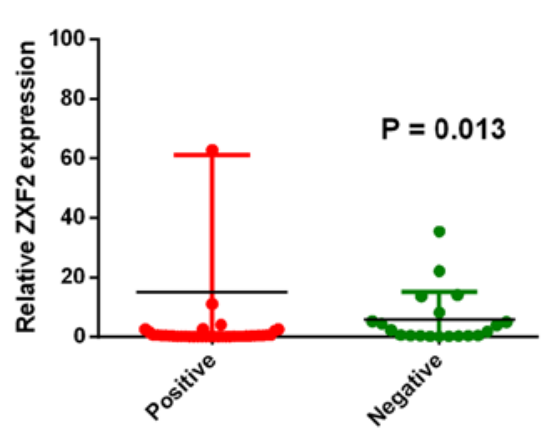

E

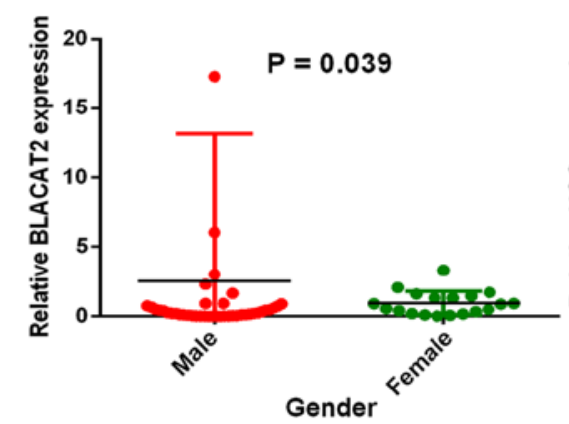

C

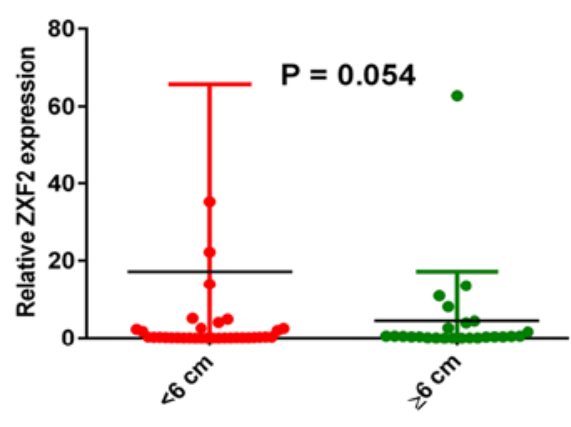

F

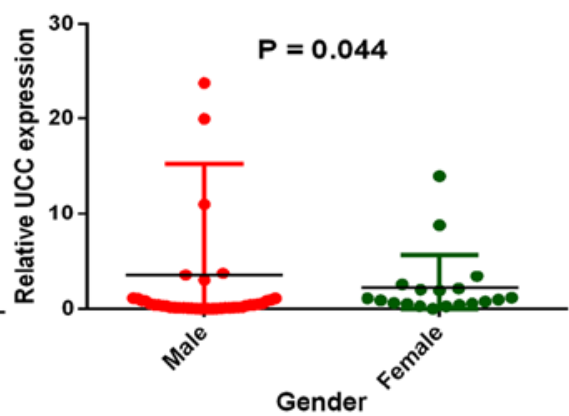

Figure 3. Associations of long non-coding RNA expression levels with the clinical characteristics of patients with GC. (A) PTPRG-AS1 expression in the Ki67-positive group ( $\mathrm{n}=41$ ) and the Ki67-negative group $(\mathrm{n}=20)$. (B) ZXF2 expression was significantly higher in the Ki67-positive group. (C) ZXF2 expression was higher in patients with smaller tumor sizes $(<6 \mathrm{~cm}$ ). (D) FOXP4-AS1 expression was significantly higher in females than males. (E) BLACAT2; and (F) UCC expressions were significantly higher in males than females. (G) UCC expression was significantly elevated in the LNM-positive group than in the LNM-negative group. Error bars represent the mean \pm SD. PTPRG-AS1, PTPRG antisense RNA 1; FOXP4-AS1, forkhead box P4 antisense RNA 1; BLACAT2, bladder cancer-associated transcript 2; UCC, upregulated in colorectal cancer; LNM, lymph node metastasis.

the clinical characteristics of patients. The characteristics that were studied include: i) Sex; ii) age; iii) lymph node metastasis status; iv) tumor grade; v) tumor size; vi) Ki67 expression status; and vii) Ki67 percentage of expression. PTPRG-AS1 expression was associated with the cell proliferation marker Ki67 ( $\mathrm{P}=0.023$; Fig. 3A). Similarly, patients in the Ki67-positive group $(n=41)$ were shown to have significantly higher ZXF2 expression than patients in the Ki67-negative group $(n=20)(P=0.013$; Fig. 3B). Both PTPRG-AS1 and ZXF2 expression levels increased with increase in the percentage of Ki67 expression. Patients with Ki67 expression levels $\geq 50 \%$ $(\mathrm{n}=24)$ had higher PTPRG-AS1 and ZXF2 expression than those with Ki67 expression levels $<50 \%(\mathrm{n}=37)$. A relationship between ZXF2 expression and tumor size was also found. Patients with tumors $<6 \mathrm{~cm}(\mathrm{n}=36)$ had increased ZXF2 expression compared with patients with tumors $\geq 6 \mathrm{~cm}(\mathrm{n}=25)$. However, this was not statistically significant
( $\mathrm{P}=0.054$; Fig. 3C). In addition, differential expression levels of FOXP4-AS1, BLACAT2 and UCC were observed between males and females. FOXP4-AS1 was significantly increased in females $(n=9)$ compared with males $(n=42)(P=0.043$; Fig. 3D). BLACAT2 expression was significantly higher in males $(n=42)$ than in females $(n=19)(P=0.037$; Fig. 3E). Similarly, UCC expression was higher in males $(n=42)$ than females $(\mathrm{n}=19)(\mathrm{P}=0.044$; Fig. 3F). Patients with lymph node metastasis $(n=46)$ had higher UCC expression than patients with no lymph node metastasis $(\mathrm{n}=15)(\mathrm{P}=0.048$; Fig. 3G), implicating UCC overexpression in GC metastasis. UCC expression was also closely associated with the expression status of cell proliferation marker Ki67, although this was not statistically significant $(\mathrm{P}=0.074)$. Collectively, these results indicate the associations of PTPRG-AS1, ZXF2, BLACAT2, FOXP4-AS1 and UCC expression levels with clinical and demographic characteristics of patients with GC. 
A

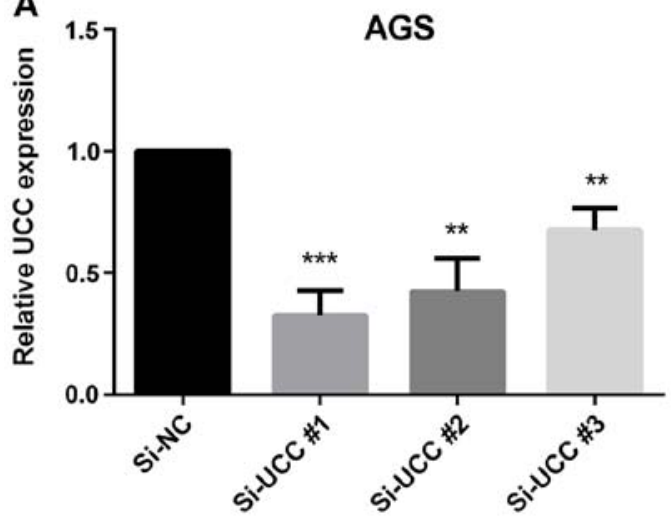

B

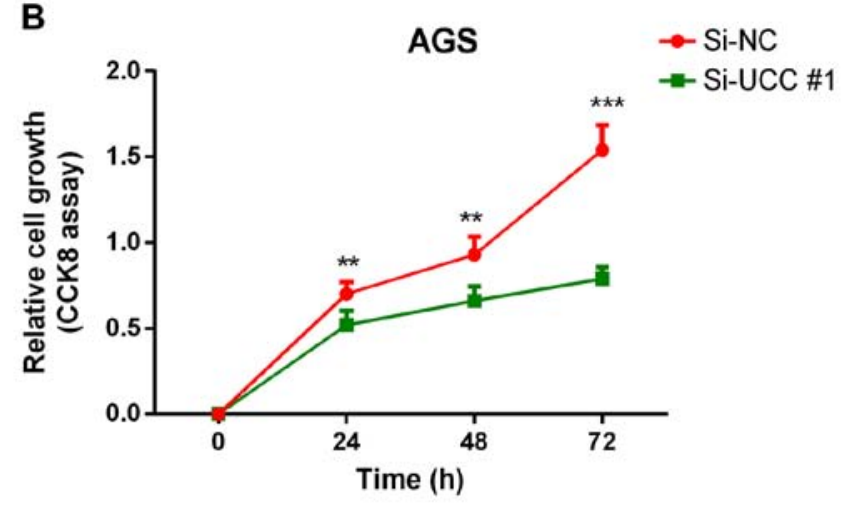

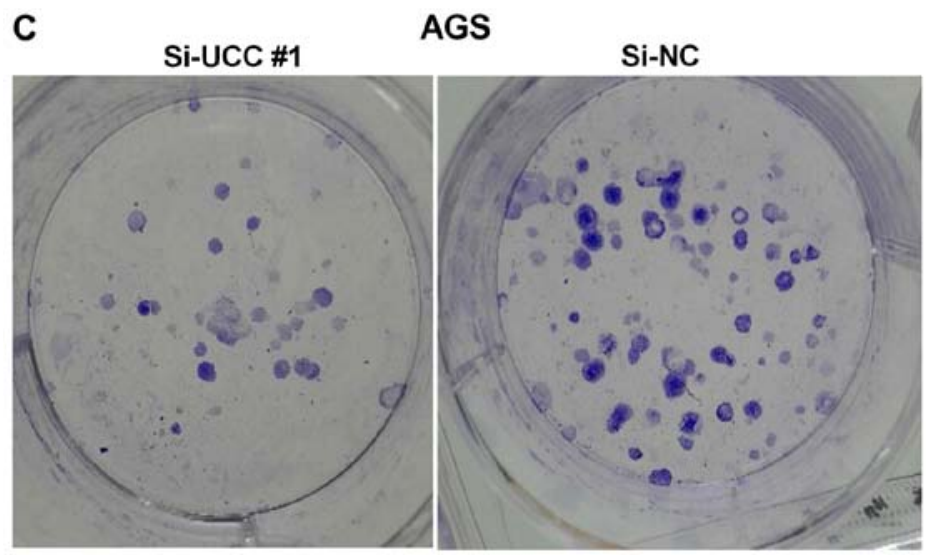

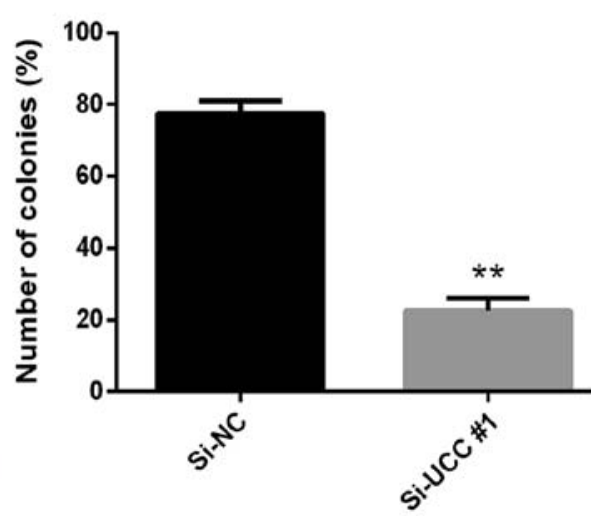

Figure 4. UCC promoted gastric cancer cell proliferation in vitro. (A) Reverse transcription-quantitative PCR results demonstrate the efficiency of UCC knockdown in AGS cells. (B) CCK-8 assay revealed that knockdown of UCC suppressed cell proliferation in AGS cells in vitro. (C) Colony formation assay revealed that knockdown of UCC reduced the number and sizes of colonies in AGS cells in vitro. ${ }^{* *} \mathrm{P}<0.01$ and ${ }^{* * *} \mathrm{P}<0.001$. Error bars represent the mean $\pm \mathrm{SD}$. UCC, upregulated in colorectal cancer; si, small interfering RNA; NC, negative control.

UCC promotes GC cell proliferation in vitro. Uncontrolled cell proliferation has been proven to be one of the hallmarks of cancer (36). Thus, the effect of one of the upregulated lncRNAs (UCC) on GC cell proliferation was investigated. Loss-of-function experiments were performed on UCC using siRNAs to ascertain its effect on GC cell proliferation. The GC cell line AGS, in which UCC expression was found to be significantly upregulated (Fig. 2E), was transfected with three siRNA oligonucleotides in order to achieve siRNA-mediated UCC knockdown. Twenty-four hours after transfection, the efficiency of transfections was verified by RT-qPCR analysis (Fig. 4A). The CCK-8 cell proliferation assay results revealed that silencing UCC markedly reduced the rate of cell proliferation (Fig. 4B). The colony formation assay results demonstrated a significant reduction in the numbers and sizes of colonies following knockdown of UCC in AGS cells $(\mathrm{P}=0.007$; Fig. 4C) indicating that UCC promotes GC cell proliferation in vitro.

Prognostic efficacy of PTPRG-AS1, FOXP4-AS1, BLACAT2, $Z X F 2$ and $U C C$ in $G C$. The prognostic efficacy of BLACAT2, FOXP4-AS1 and UCC was assessed using the GEPIA web server (http://gepia.cancer-pku.cn), whereas the prognostic efficacy of PTPRG-AS1 was examined using the Kaplan Meier Plot database (www.kmplot.com) (Affymetrix ID: 232242_at). High FOXP4-AS1 expression was significantly associated with shorter disease-free survival (DFS) rates compared with low FOXP4-AS1 expression ( $\mathrm{P}=0.023$; Fig. 5A). No significant associations were found between increased expression of BLACAT2 and UCC and the OS and DFS rates of patients with GC (Fig. 5B and C). However, high PTPRG-AS1 was significantly associated with poor $\mathrm{OS}$ rates in all patients $(\mathrm{P}=0.019$; Fig. 6A), male patients alone ( $\mathrm{P}=0.041$; Fig. 6B), female patients alone $(\mathrm{P}=0.040$; Fig. $6 \mathrm{C})$, patients treated with surgery alone $(\mathrm{P}=0.047$; Fig. 6D), patients in clinical stage $3(\mathrm{P}=0.0026$; Fig. 6E), HER2- patients ( $\mathrm{P}=0.024$; Fig. $6 \mathrm{~F})$, patients with stage M0 GC and no metastasis ( $\mathrm{P}=0.017$; Fig. 6G), patients with stage N0 GC and no lymph node metastasis $(\mathrm{P}=0.040$; Fig. $6 \mathrm{H}$ ) and patients with stage N1 GC and few lymph node metastasis sites ( $\mathrm{P}=0.006$; Fig. 6I). Shorter survival rates were also observed among patients treated with 5-fluorouracil adjuvant alone who had high PTPRG-AS1 expression compared with patients with low PTPRG-AS1 expression, although this was not statistically significant $(\mathrm{P}=0.45$; Table II). Correlation analyses were also performed on other stages, classifications and pathological grades. The results, which are summarized in Table II, demonstrate the prognostic efficacy of the lncRNAs in patients with $\mathrm{GC}$ that have different clinicopathological characteristics.

Genetic alteration of BLACAT2 is a marker of poor outcome for patients with GC. Genetic alterations of the lncRNAs in 
A

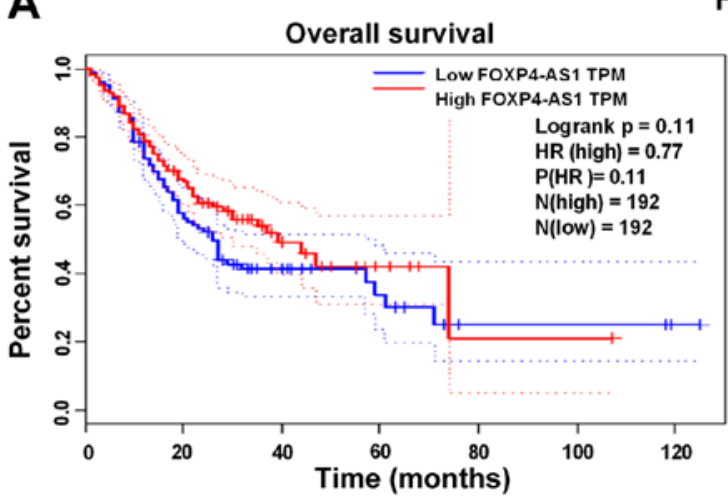

FOXP4-AS1

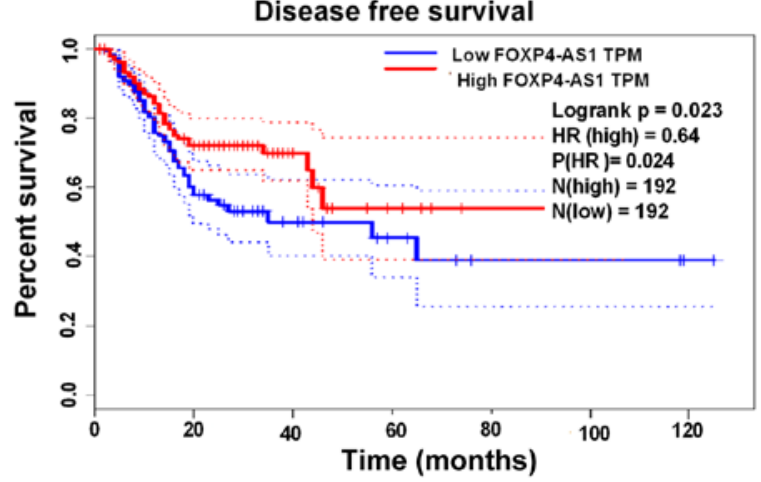

B

BLACAT2 (LINC00958)
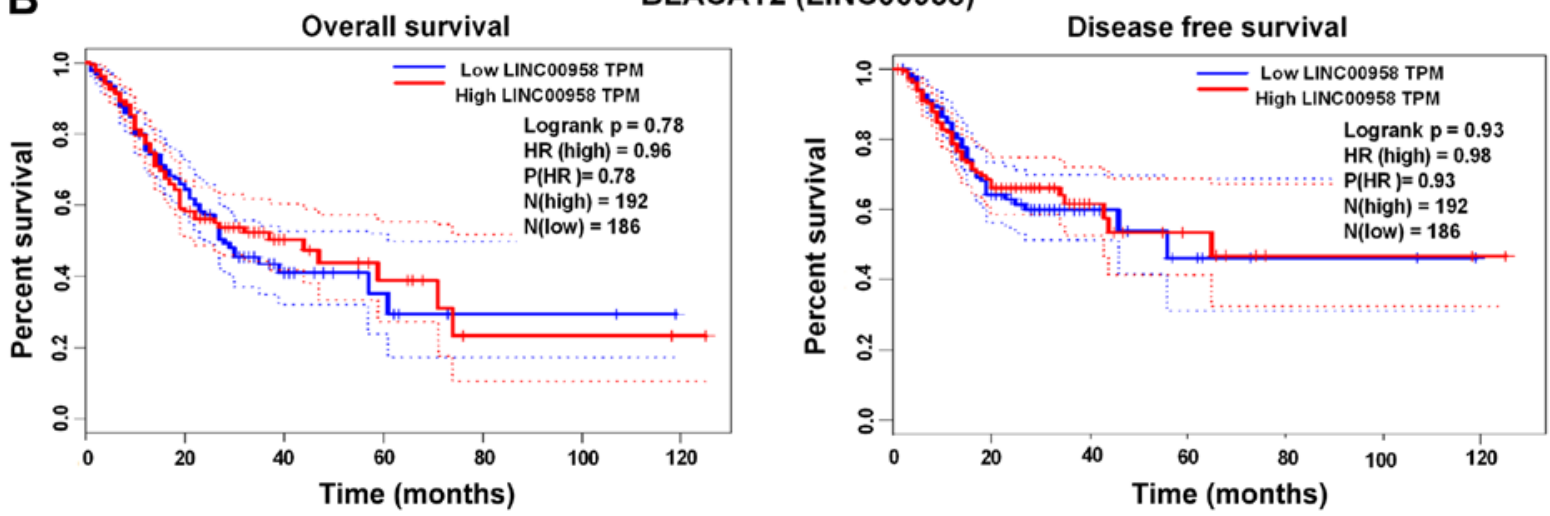

C

UCC (CTD-2227E11.1)
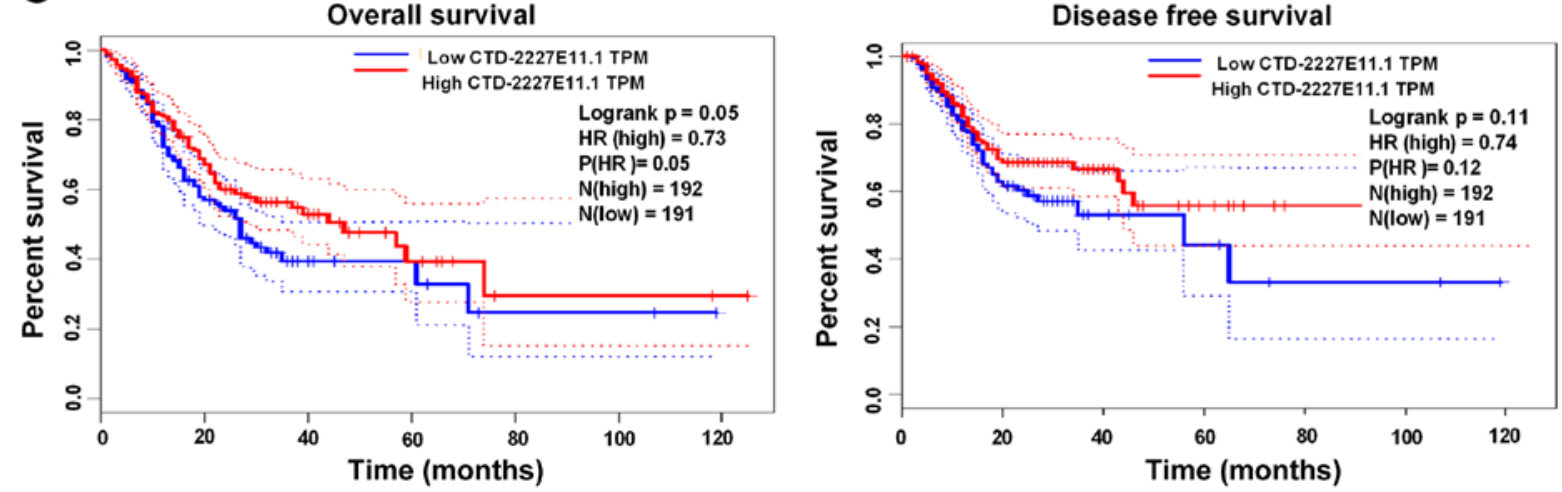

Figure 5. Prognostic efficacy of FOXP4-AS1, BLACAT2 and UCC in GC evaluated using GEPIA. (A) Kaplan-Meier survival curves revealed that high FOXP4-AS1 expression significantly correlated with shorter DFS survival times of patients. (B) BLACAT2 expression levels were not significantly associated with GC prognosis. (C) UCC expression levels were not significantly associated with GC prognosis. GEPIA, Gene Expression Profiling Interactive Analysis; FOXP4-AS1, forkhead box P4 antisense RNA 1; BLACAT2, bladder cancer-associated transcript 2; UCC, upregulated in colorectal cancer.

GC were analyzed using the cBioPortal online tool. A total of 1,365 patients from six datasets of patients with GC were evaluated. No alterations were found in the FOXP4-AS1, ZXF2 and UCC genes in GC. Regarding PTPRG-AS1, alterations ranging from 1.82 to $2.37 \%$ were found for the gene sets submitted for analysis (Fig. 7A). The percentage of genetic alterations in PTPRG-AS1 for GC was 1.1\% (Fig. 7B). Following cBioPortal and Kaplan-Meier plotter analyses and a log-rank test, the results indicated that there was no significant difference in OS and DFS in patients with or without alterations in PTPRG-AS1 $(\mathrm{P}=0.161$ and $\mathrm{P}=0.479$ respectively; Fig. 7C). Regarding the BLACAT2 gene, alterations were found ranging from 0.21 to $0.34 \%$ (Fig. 8A) and the percentage of genetic alterations was $0.2 \%$ (Fig. 8B).
Following cBioPortal and Kaplan-Meier plotter analyses and a log-rank test, the results indicated that there was no significant difference in OS in cases with or without alterations in BLACAT2 $(\mathrm{P}=0.239)$. By contrast, cases with alterations in BLACAT2 had significantly poorer DFS rates than those without alterations ( $\mathrm{P}=0.0023$; Fig. 8C).

\section{Discussion}

Therapeutic regimens available for GC, including trastuzumab, a widely administered drug, have obvious limitations, including their inability to be used for a wider range of patients (22). Therefore, GC remains one of the leading causes of cancer-related deaths (37). Studies have shown that 
Table II. Correlation of high PTPRG antisense RNA 1 expression with other clinical characteristics of patients with gastric cancer.

\begin{tabular}{|c|c|c|c|}
\hline Characteristics & $\mathrm{n}$ & HR 95\% CI & P-value \\
\hline \multicolumn{4}{|l|}{ Clinical stages } \\
\hline 2 & 126 & $1.7(0.89-3.26$ & 0.11 \\
\hline 4 & 140 & $0.72(0.46-1.11)$ & 0.13 \\
\hline \multicolumn{4}{|l|}{ Pathological grades } \\
\hline Poorly differentiated & 121 & $1.26(0.78-2.06)$ & 0.34 \\
\hline Moderately differentiated & 67 & $0.47(0.24-0.92)$ & 0.024 \\
\hline \multicolumn{4}{|l|}{ Well differentiated } \\
\hline \multicolumn{4}{|l|}{ HER2 status } \\
\hline Positive & 202 & $1.43(0.94-2.19)$ & 0.097 \\
\hline \multicolumn{4}{|l|}{ Treatments } \\
\hline 5-FU based adjuvant & 34 & $1.42(0.57-3.54)$ & 0.45 \\
\hline Other adjuvants (e.g. irinotecan) & 76 & $0.52(0.2-1.36)$ & 0.17 \\
\hline \multicolumn{4}{|l|}{ T stage } \\
\hline 2 & 241 & $1.55(0.99-2.44)$ & 0.054 \\
\hline 4 & 38 & $0.61(0.26-1.41)$ & 0.24 \\
\hline \multicolumn{4}{|l|}{$\mathrm{N}$ stage } \\
\hline $1+2+3$ & 422 & $1.3(0.97-1.73)$ & 0.074 \\
\hline 2 & 121 & $0.71(0.45-1.11)$ & 0.13 \\
\hline 3 & 76 & $1.31(0.76-2.26)$ & 0.33 \\
\hline \multicolumn{4}{|l|}{ M stage } \\
\hline M1 & 56 & $0.63(0.33-1.22)$ & 0.17 \\
\hline \multicolumn{4}{|l|}{ Lauren classification } \\
\hline Intestinal & 269 & $1.47(0.97-2.23)$ & 0.067 \\
\hline Mixed & 29 & $1.56(0.5-4.77)$ & 0.43 \\
\hline Diffuse & 240 & $1.35(0.96-1.9)$ & 0.084 \\
\hline
\end{tabular}

CI, confidence interval; HER2, human epidermal growth factor receptor 2; HR, hazard ratio; 5-FU, 5-fluorouracil.

upregulated lncRNAs such as OCC-1, AK126698, SChLAP1, BORG and DANCR are associated with poor prognosis in cancer patients $(9,10,12-14)$. Poor prognoses may also be attributed to late diagnosis, lack of specific biomarkers and the metastatic and proliferative capacity of cancer cells $(38,39)$. Since specific biomarkers of GC are currently limited, patients are more often diagnosed at a later, usually metastatic stage. This increases the risk of disease recurrence, GC-related deaths and poor prognoses. Consequently, there is a necessity for further exploration of the genetic and molecular modifications that characterize gastric carcinogenesis and an urgent need for the identification of highly sensitive and specific biomarkers.

In the present study, the expression profiles, functions and prognostic efficacy of five lncRNAs in GC, specifically PTPRG-AS1, FOXP4-AS1, BLACAT2, ZXF2 and UCC, were investigated. Expression analyses revealed that all five lncRNAs were upregulated in tumor tissues relative to ANTs, thus indicating differential expression patterns of the lncRNAs between cancer and healthy tissues. This was a significant finding since the aberrant expression patterns of the lncRNAs suggest their application as diagnostic markers for GC at the molecular level. The present study results regarding the upregulation of PTPRG-AS1, FOXP4-AS1, BLACAT2, ZXF2 and UCC are in accordance with the results of Iranpour et al (23), Li et al (25), He et al (26), Yang et al (27) and Huang et al (15), which conducted studies on breast, colorectal, bladder, lung and colorectal cancers, respectively. The results of the current study were further verified in four GC cell lines. As expected, all five lncRNAs were highly expressed in GC cell lines relative to the healthy gastric epithelial cell line GES-1. Significant associations were found between BLACAT2, FOXP4-AS1, UCC and the sex of patients with GC. BLACAT2 and UCC expression levels were higher in males than in females. Nonetheless, higher FOXP4-AS1 expression was observed in females than in males. Therefore, it was concluded that sex may be an important predisposing factor for GC. Biological factors, including age and sex, have been associated with the development of certain cancer types and the sex differential in susceptibility may provide insight into the etiology of cancer (40). The present study also reported new findings on the relationship between BLACAT2, FOXP4-AS1 and UCC expression and sex predisposition to GC. 
A
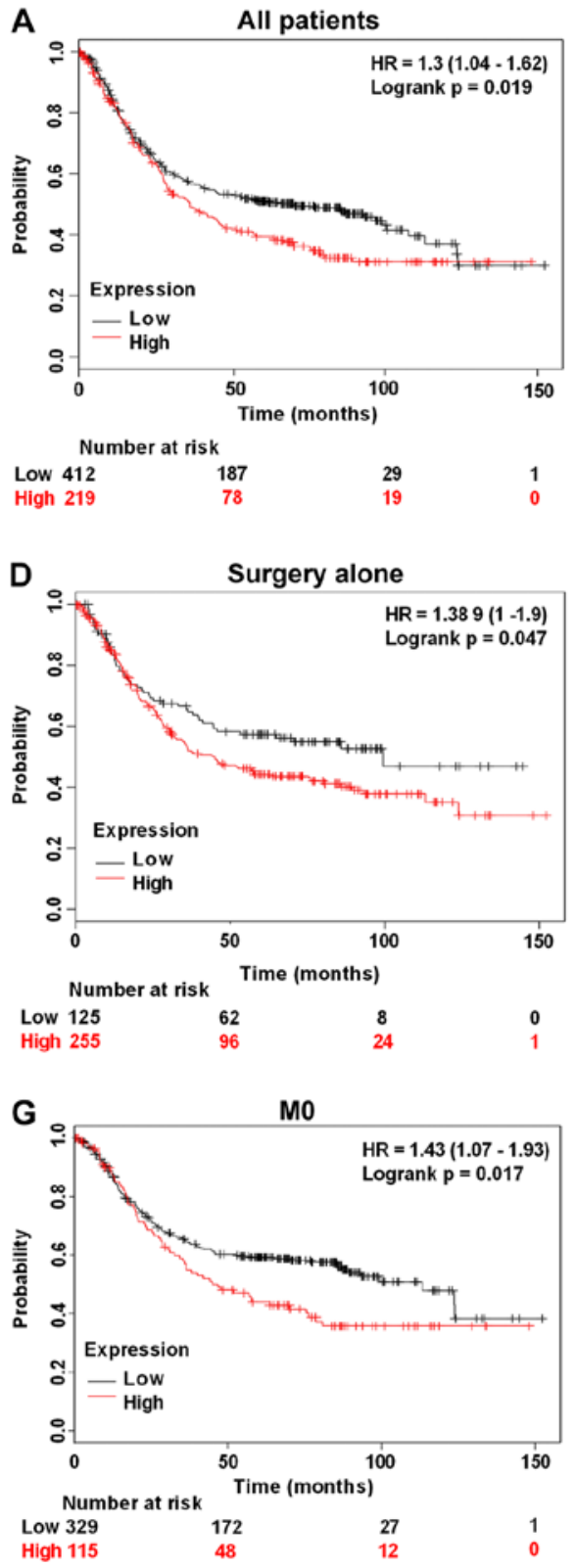

B

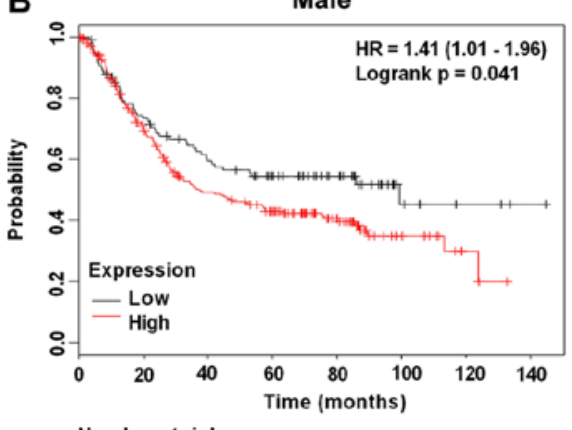

Number at risk

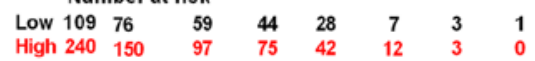

E

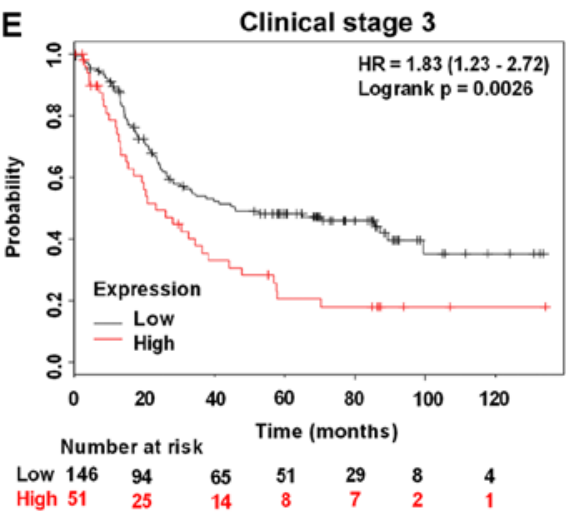

$\mathrm{H}$

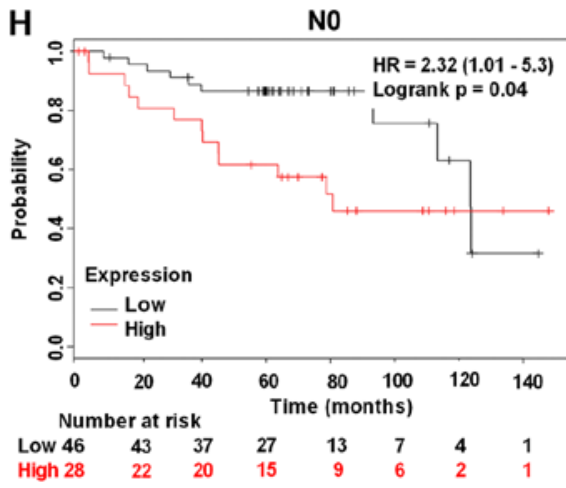

C

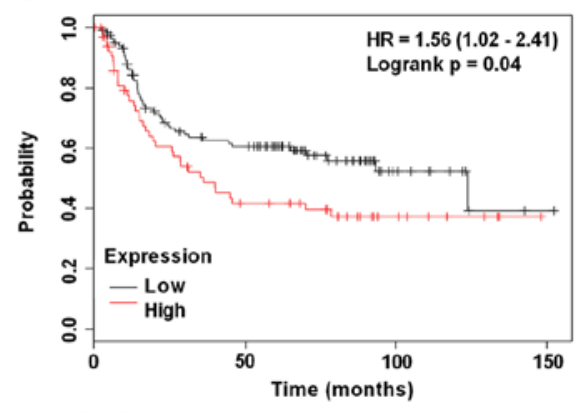

Number at risk

$\begin{array}{llcl}\text { Low } 120 & 60 & 11 & 1 \\ \text { High } 67 & 23 & 8 & 0\end{array}$

F

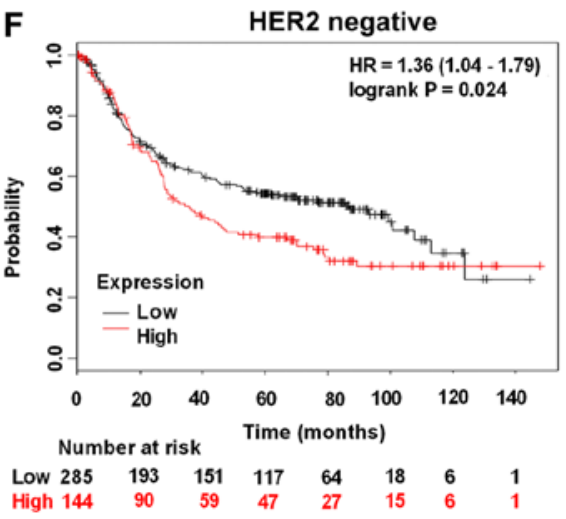

I

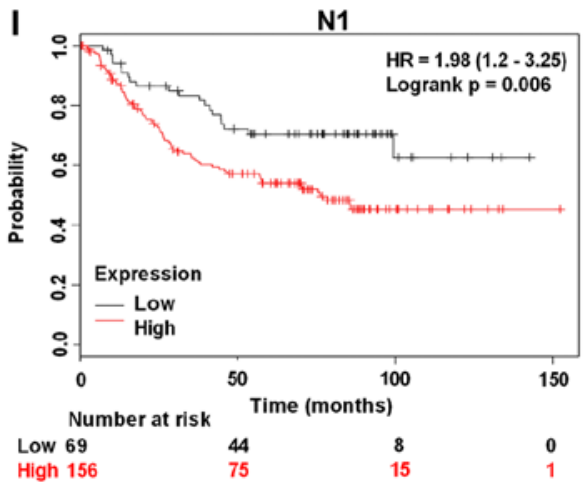

Figure 6. Prognostic efficacy of PTPRG-AS1 in GC evaluated using the Kaplan Meier Plotter. Kaplan-Meier survival curves revealed that poor OS of (A) all patients, (B) male patients, (C) female patients, (D) patients treated with surgery alone, (E) patients in clinical stage 3, (F) HER2- patients, (G) patients with no metastasis (M0), (H) patients with no lymph node metastasis (N0), and (I) patients with few lymph node metastasis sites (N1) was associated with high expression of PTPRG-AS1. PTPRG-AS1, PTPRG antisense RNA 1.

PTPRG-AS1 expression was significantly associated with the expression status of the cell proliferation marker Ki67. In particular, the expression of ZXF2 was higher in Ki67-positive patients than in Ki67-negative patients. Both PTPRG-AS1 and ZXF2 expression levels were found to increase in response to increased Ki67 expression, hence indicating that the proliferative ability of GC cells increases when the expression of PTPRG-AS1 and ZXF2 is upregulated. These results suggest the involvement of PTPRG-AS1 and ZXF2 in GC cell proliferation. As in other types of cancer, GC develops due to genetic alterations that result in generalized loss of growth control, which in turn leads to continual unregulated proliferation of cancer cells (36). Consequently, it was hypothesized that lncRNAs PTPRG-AS1 and ZXF2 are oncogenes that promote gastric tumorigenesis and/or progression by promoting GC proliferation. This hypothesis was corroborated by the findings of Iranpour et al (23) and Yang et al (27), which suggested the implication of PTPRG-AS1 and ZXF2 in the oncogenicity of breast and lung cancer, respectively. Patients with smaller tumors exhibited considerably higher ZXF2 expression than patients with tumors of a larger size, though the difference was not significant. Nevertheless, ZXF2 may be a marker of the early stages of gastric carcinogenesis. This possibility requires further investigation in large-scale studies.

The human body comprises a network of lymphatic vessels and lymph nodes mainly designed to serve protective roles against infections by filtering harmful substances (41). LNM is defined as the spread of cancer cells from a primary (original) tumor to the lymph nodes. It is a well-known and clinically accepted strong prognostic factor for the recurrence 


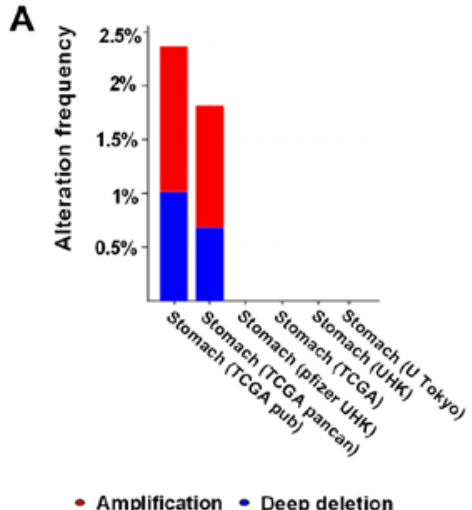

B

Study of origin

Profiled for copy number alterations

Profiled for mutations

PTPRG-AS1

Genetic alteration

Study of origin

Profiled for copy number alterations

Profiled for mutations

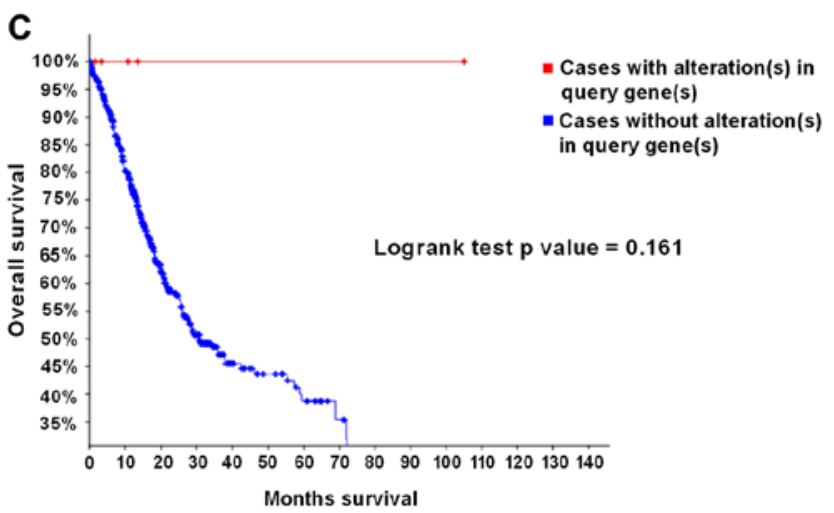
I Amplification Deep deletion || No alterations - Not profiled

Yes No

Yes No
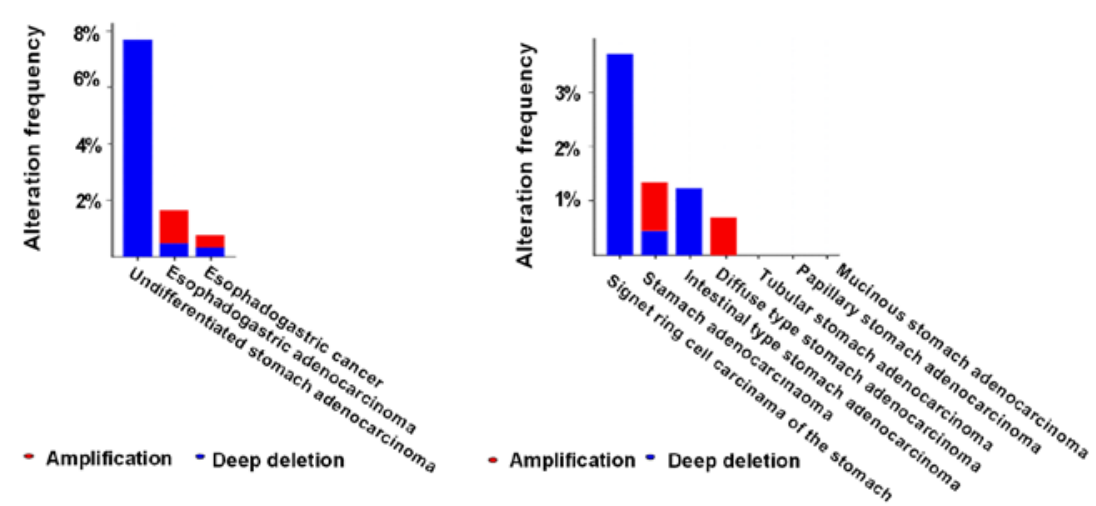

|||||||||||||||||||||||||||||||||||||||||||||||||||||||||||||||||||||||||||||||||||||| |||||||||||||| |||||||||||||||||||||||||||||||||||||||||||||| |- ||||||||||||||||||||||||||||||||||||||||| 1.1\% I IIIIIIIII

IStomach adenocarcinoma (ptizer and UHK, nat. genet 2014) 』 Stomach adenocarcinoma (TCGA, nature 2014)

| Stomach adenocarcinoma (TCGA, pancancer atlas) \Stomach adenocarcinoma (TCGA, provisional)

| Stomach adenocarcinoma (U Tokyo, nat. genet 2014) \Stomach adenocarcinoma (UHK, nat. genet 2011)

Figure 7. Alteration frequency of PTPRG-AS1 gene in gastric cancer identified using cBioPortal. (A) Summary of alterations in PTPRG-AS1. (B) Oncoprint visual summary of alteration on a query of PTPRG-AS1. (C) Kaplan-Meier plots comparing overall survival and disease-free survival in cases with/without PTPRG-AS1 gene alterations. PTPRG-AS1, PTPRG antisense RNA 1; TCGA, The Cancer Genome Atlas.

and survival of patients with most solid tumors (41). Previous findings have demonstrated the involvement of the unique lymph node microenvironment in the formation of systemic metastasis suggesting LNM as a source of further cancer spread and poor prognosis. Adequate management of LNM could reduce further systemic metastasis and improve patient outcomes (42). In the current study, a higher UCC expression was observed in patients with LNM than in those without LNM. This association between UCC expression and LNM suggests that the overexpression of UCC may be responsible for metastatic competence and disease progression in patients with GC. This is an indication of the role of UCC in GC metastasis and is in consonance with an aforementioned study suggesting UCC upregulation in the metastasis of colorectal cancer cells (15).

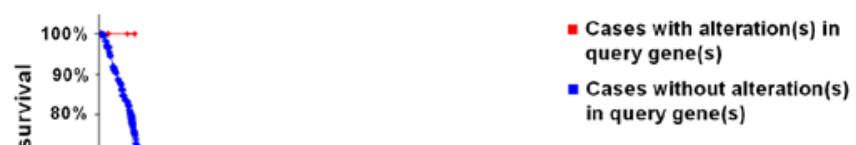

Logrank test $p$ value 0.479

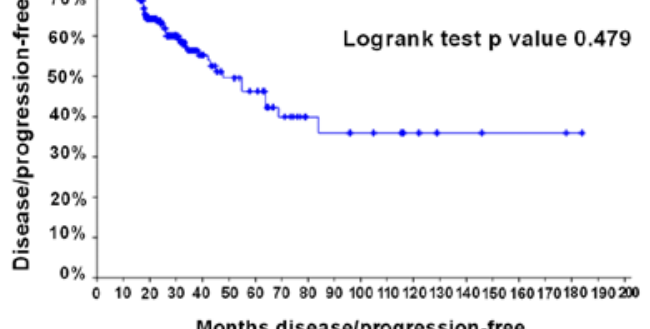

Months disease/progression-free query gene(s)

in query gene(s)
According to the CCK-8 assay results, UCC was identified as a key driver of GC growth. Knockdown of UCC significantly inhibited cell proliferation in AGS cells. This finding was further confirmed by performing a colony formation assay. In comparison with controls, the number and sizes of GC cell colonies were found to be significantly reduced after UCC knockdown, implicating UCC in GC cell proliferation. Similar findings were reported in an aforementioned study on colorectal cancer (15).

The prolonged survival times observed among patients with high FOXP4-AS1 expression in the present study suggest that FOXP4-AS1 is a marker of favorable outcome for patients with GC. No associations were identified between high expression of BLACAT2 or UCC with the OS or DFS of patients. This finding requires further validation in studies with larger 

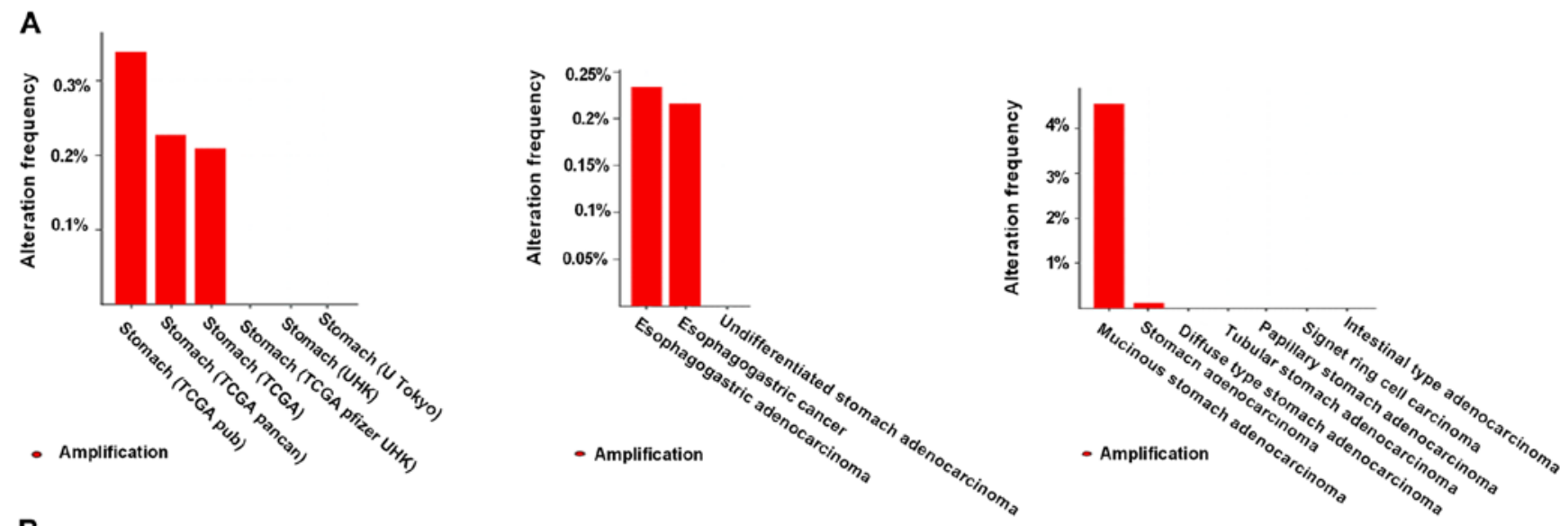

\section{B}

Study of origin

Profiled for copy number alterations

Profiled for mutations

LINC00958

Genetic alteration

Study of origin

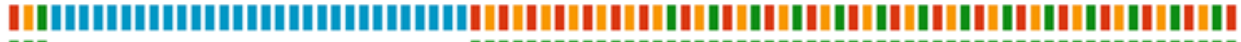
III-

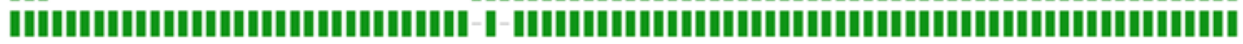

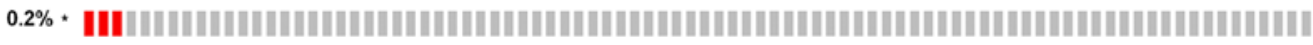

| Amplification || No alterations - Not profiled

| Stomach adenocarcinoma (pfizer and UHK, nat. genet 2014) | Stomach adenocarcinoma (TCGA, nature 2014)

|| Stomach adenocarcinoma (TCGA pancancer atlas) | Stomach adenocarcinoma (TCGA, provisional)

| Stomach adenocarcinoma (U Tokyo, nat. genet 2014) | Stomach adenocarcinoma (UHK, nat. genet 2011)

Profiled for copy number

alterations

I Yes - No

Profiled for mutations

Ies No

C

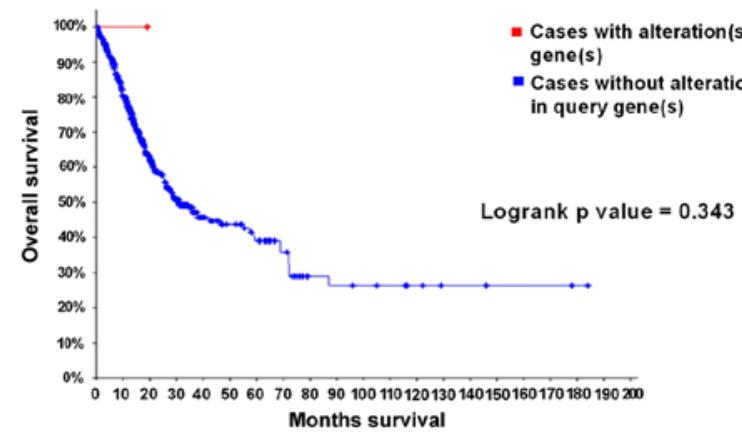

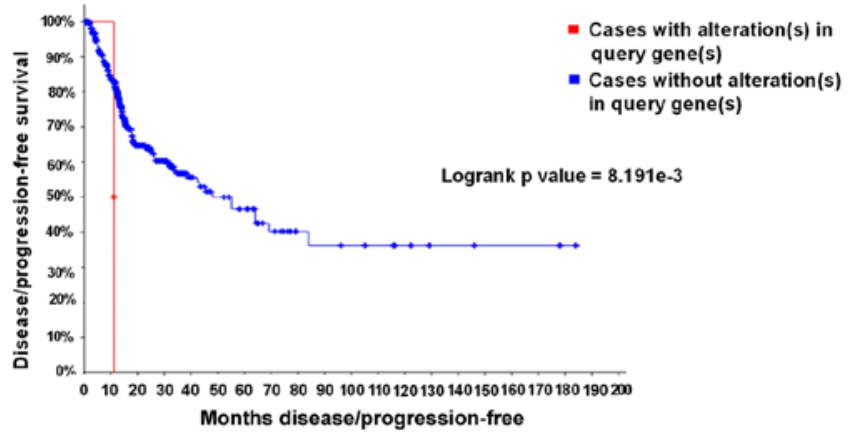

Figure 8. Alteration frequency of BLACAT2 (LINC00958) gene in gastric cancer identified using cBioPortal. (A) Summary of alterations in BLACAT2. (B) Oncoprint visual summary of alteration on a query of BLACAT2. (C) Kaplan-Meier plots comparing overall survival and disease-free survival in cases with/without BLACAT2 gene alterations. BLACAT2, bladder cancer-associated transcript 2; TCGA, The Cancer Genome Atlas.

sample sizes. However, high expression of PTPRG-AS1 was significantly associated with poor OS probability in all patients; those with high expression of PTPRG-AS1 had shorter survival rates than those with low PTPRG-AS1 expression. PTPRG-AS1 may be a novel predictor of prognosis for patients with GC.

Surgical resection is usually the first line of treatment for patients with GC. Thus far, it is regarded as the only reliable possibility of a curative treatment and the only effective therapy, though the locoregional recurrence and five-year survival rates following resection remain poor (43). Shorter survival rates were observed among patients with high PTPRG-AS1 expression treated with surgery alone or 5-FU adjuvant alone than in those with low PTPRG-AS1 expression subjected to the same treatment. It is possible that PTPRG-AS1 upregulation may be responsible for resistance to GC therapy.
Over $80 \%$ of patients with GC are HER2- (44). Although positive HER2 status is generally associated with poor prognosis for patients with $\mathrm{GC}$, a recent study revealed that, when treated with trastuzumab, HER $2^{+}$patients had better prognosis than HER2- patients (44). Therefore, the development of targeted therapies for HER2- patients may prove to be beneficial. The results from the survival analysis performed in the current study showed that a high expression of PTPRG-AS1 was associated with poor prognosis for HER2- patients, as OS significantly decreased in patients with a high expression of this lncRNA compared with those that had a low expression. PTPRG-AS1 may be a novel therapeutic target for HER2patients especially since the trastuzumab regimen has been found to be ineffective in these patients (22).

Of note, patients with advanced GC stage (stage III), patients with earlier non-metastatic stages of GC (stage M0) and no lymph node metastasis (stage N0) and those with metastasis in 
few lymph nodes (stage N1) and high PTPRG-AS1 expression in the current study, had poorer survival rates compared with patients that had low expression. Poorer survival rates were also observed among patients with other pathological stages of GC that had a high PTPRG-AS1 expression compared with those that had downregulated expression. Thus, it was concluded that PTPRG-AS1 may be a novel prognostic predictor of both early and advanced GC and may serve as a marker of patient outcomes and a therapeutic target for patients with different pathological stages and subgroups of GC.

Mutations and gene amplifications are linked to carcinogenesis; mutant genes cause a gain-of-function phenotype, are involved in tumorigenesis and related to patient outcomes (45). Herein, the percentages of alterations in PTPRG-AS1 and BLACAT2 among patients were identified as 1.1 and $0.2 \%$, respectively. There were no significant differences in OS and DFS in cases with or without alterations in PTPRG-AS1 and no significant difference in OS rates in cases with or without alterations in BLACAT2. However, cases with this type of alteration had significantly poorer DFS rates than those without alterations, indicating that BLACAT2 gene mutations are associated with poor prognosis in GC. Upregulated lncRNAs may be more readily applied as biomarkers than downregulated lncRNAs (15). A number of studies have reported an association between IncRNA upregulation and patient outcomes and have suggested their application as cancer biomarkers. For example, Gao et al (46) found that plasma orosomucoid 2 was significantly upregulated in patients with stage II colorectal cancer and may be used as a biomarker in diagnosis and prognostic evaluation. Li et al (47) revealed that high MALAT1 expression was associated with decreased survival and poor response of advanced colorectal cancer patients to oxaliplatin-based chemotherapy. Du et al (48) demonstrated that increased expression of urinary uc004cox.4 was significantly associated with poorer prognosis in terms of recurrence-free survival of non-muscle invasive bladder cancer and may be combined with future non-invasive biomarkers for the diagnosis and prognosis of bladder cancer, provided that it is validated in a large-scale study. According to Li et al (49), a high expression of SPINT1-AS1 was associated with poor clinical outcome and SPINT1-AS1 may be a promising biomarker for the prognostic prediction and treatment monitoring of patients with colorectal cancer. Moreover, the present study reported new findings on the expression patterns, functions and clinical significance of PTPRG-AS1, FOXP4-AS1, BLACAT2, ZXF2 and UCC and their potential for application as diagnostic, prognostic and therapeutic targets in GC.

Determination of target RNAs or proteins that lncRNAs interact with is regarded as an important step in characterizing the functions of IncRNAs (50). A recent study demonstrated that IncRNAs regulate inflammation and reparative responses by competitively sponging miRNAs and acting as competing endogenous RNAs (51). Identification of interactions and complex regulatory networks of IncRNAs, miRNAs and other relevant genes may provide insight into the molecular mechanisms involved in human cancer and the development of diagnostic and therapeutic strategies (52). Certain molecular interactions of the lncRNAs in our study have also been reported by a number of studies. For instance, BLACAT2 was found to mediate the interaction of WD repeat domain 5 (WDR5), a core component of the histone $\mathrm{H} 3 \mathrm{~K} 4$ methyltransferase complex that is required for vertebrate development, with multiple promoter target sequences of vascular endothelial growth factor C (26). WDR5 is involved in the self-renewal of pluripotent stem cells and cell differentiation, and interactions with WDR 5 may induce tumorigenesis by promoting target gene recognition (53). By contrast, UCC was identified as a possible target of miR-143, which has been found to be downregulated in a variety of cancer types (15). ZXF2 has been reported to interact with c-myc and enhance lung cancer progression via the c-myc/e-cadherin pathway (27) and PTPRG-AS1 has been suggested to downregulate the expression of PTPRG, a tumor suppressor gene (24), thereby enhancing disease progression in breast cancer. The results from these studies confirm the proto-oncogenic involvements of the studied lncRNAs in various malignancies. However, their precise molecular interactions in GC need to be investigated.

The present study had certain limitations. First, the number of GC tissue samples used was relatively small. Although significantly different expression levels of PTPRG-AS1, FOXP4-AS1, BLACAT2 and UCC were observed, ZXF2 upregulation was not statistically significant, probably due to this limitation. Secondly, access to the follow-up data of patients was not available, because the samples were collected from patients with newly diagnosed GC at the time of analysis; consequently, the prognostic efficacy of the lncRNAs could not be determined using the results of RT-qPCR analysis. However, the effects of high lncRNA expression on patient outcomes were examined using data from publicly available online databases. Despite the above shortcomings, the present study revealed that PTPRG-AS1, FOXP4-AS1, BLACAT2, ZXF2 and UCC are upregulated in GC and have the potential for application as biomarkers for GC detection at the molecular level. The involvement of PTPRG-AS1 and ZXF2 in GC growth was also revealed and ZXF2 was identified as a potential marker of the early stages of gastric carcinogenesis. Furthermore, associations were observed between BLACAT2, FOXP4-AS1, UCC and sex predisposition to GC. An association was also identified between UCC overexpression and lymph node metastasis and its role in promoting GC cell proliferation in vitro. Finally, it was shown that BLACAT2 gene alterations were associated with worse DFS outcome and PTPRG-AS1 overexpression was associated with worse OS outcome for all patients with GC and the respective subgroups of GC patients. Therefore, these lncRNAs are promising novel prognostic predictors and therapeutic targets for different categories of patients with GC.

In conclusion, PTPRG-AS1, FOXP4-AS1, BLACAT2, ZXF2 and UCC are potential biomarkers for GC detection at the molecular level and they may be individually utilized as therapeutic targets for GC and for prognostic predictions. Nonetheless, further in vitro and in vivo research is required for each lncRNA in order to ascertain their specific involvements in gastric carcinogenesis.

\section{Acknowledgements}

The authors would like to acknowledge The Second Hospital of Shandong University for supporting the study and providing data. 


\section{Funding}

This work was supported by the Fundamental Research Funds of Shandong University (grant nos. 2017BTS01, 2018JC002 and 2017JC031) and the Key Research and Development Program of Shandong Province (grant no. 2018FYJH0505).

\section{Availability of data and materials}

The datasets used and analyzed in the present study are available from the corresponding author upon reasonable request.

\section{Authors' contributions}

HBB, YSW, LD and CW designed the study. HBB performed the experiments and wrote the manuscript. $\mathrm{HBB}$ and MAT analyzed and interpreted the data. HBB, MAT and AGAN revised the paper. SS, NL and AGAN assisted with experimental protocols. YSW, LD and CW supervised the study. All authors approved the final version of the manuscript.

\section{Ethics approval and consent to participate}

The present study was approved by the Ethics Committee of The Second Hospital of Shandong University. All experimental procedures were conducted in accordance with the Declaration of Helsinki.

\section{Patients consent for publication}

Not applicable.

\section{Competing interests}

The authors declare that they have no competing interests.

\section{References}

1. Xu MD, Wang Y, Weng W, Wei P, Qi P, Zhang Q, Tan C, Ni SJ, Dong L, Yang Y, et al: A positive feedback loop of lncRNA-PVT1 and FOXM1 facilitates gastric cancer growth and invasion. Clin Cancer Res 23: 2071-2080, 2017.

2. Zhao Y, Liu Y, Lin L, Huang Q, He W, Zhang S, Dong S, Wen Z, Rao J, Liao W and Shi M: The lncRNA MACC1-AS1 promotes gastric cancer cell metabolic plasticity via AMPK/Lin28 mediated mRNA stability of MACC1. Mol Cancer 17: 69, 2018.

3. Zhang K, Shi H, Xi H, Wu X, Cui J, Gao Y, Liang W, Hu C, Liu Y, Li J, et al: Genome-wide lncRNA microarray profiling identifies novel circulating lncRNAs for detection of gastric cancer. Theranostics 7: 213-227, 2017

4. Xia H, Chen Q, Chen Y, Ge X, Leng W, Tang Q, Ren M, Chen L, Yuan D,Zhang Y, et al: The IncRNA MALAT1 is a novel biomarker for gastric cancer metastasis. Oncotarget 7: 56209-56218, 2016.

5. Lv J, Qiu M, Xia W, Liu C, Xu Y, Wang J, Leng X, Huang S, Zhu R, Zhao M, et al: High expression of long non-coding RNA SBF2-AS1 promotes proliferation in non-small cell lung cancer. J Exp Clin Cancer Res 35: 75, 2016.

6. Zhang Y, Song X, Wang X, Hu J and Jiang L: Silencing of LncRNA HULC enhances chemotherapy induced apoptosis in human gastric cancer. J Med Biochem 35: 137-143, 2016.

7. Gu Y, Chen T, Li G, Yu X, Lu Y, Wang H and Teng L: LncRNAs: Emerging biomarkers in gastric cancer. Future Oncol 11: 2427-2441, 2015.

8. Wu H, Hu Y, Liu X, Song W, Gong P, Zhang K, Chen Z, Zhou M, Shen X, Qian Y and Fan H: LncRNA TRERNA1 function as an enhancer of SNAI1 promotes gastric cancer metastasis by regulating epithelial-mesenchymal transition. Mol Ther Nucleic Acids 8: 291-299, 2017.
9. Lan Y, Xiao X, He Z, Luo Y, Wu C, Li L and Song X: Long noncoding RNA OCC-1 suppresses cell growth through destabilizing HuR protein in colorectal cancer. Nucleic Acids Res 46: 5809-5821, 2018

10. Fu X, Li H, Liu C, Hu B, Li T and Wang Y: Long noncoding RNA AK126698 inhibits proliferation and migration of non-small cell lung cancer cells by targeting Frizzled- 8 and suppressing Wnt/ $\beta$-catenin signaling pathway. Onco Targets Ther 9: 3815-3827, 2016.

11. Zhang S, Zhong G, He W, Yu H, Huang J and Lin T: IncRNA Up-regulated in nonmuscle invasive bladder cancer facilitates tumor growth and acts as a negative prognostic factor of recurrence. J Urol 196: 1270-1278, 2016.

12. Gooding AJ, Zhang B, Jahanbani FK, Gilmore HL, Chang JC, Valadkhan S and Schiemann WP: The lncRNA BORG drives breast cancer metastasis and disease recurrence. Sci Rep 7: 12698, 2017.

13. Prensner JR, Iyer MK, Sahu A, Asangani IA, Cao Q, Patel L, Vergara IA, Davicioni E, Erho N, Ghadessi M, et al: The long noncoding RNA SChLAP1 promotes aggressive prostate cancer and antagonizes the SWI/SNF complex. Nat Genet 45: 1392-1398, 2013.

14. Mao Z, Li H, Du B, Cui K, Xing Y, Zhao X and Zai S: LncRNA DANCR promotes migration and invasion through suppression of lncRNA-LET in gastric cancer cells. Biosci Rep 37: BSR20171070, 2017.

15. Huang FT, Chen WY, Gu ZQ, Zhuang YY, Li CQ, Wang LY, Peng JF, Zhu Z, Luo X, Li YH, et al: The novel long intergenic noncoding RNA UCC promotes colorectal cancer progression by sponging miR-143. Cell Death Dis 8: e2778, 2017.

16. Zhao J, Du P, Cui P, Qin Y, Hu C, Wu J, Zhou Z, Zhang W, Qin L and Huang G: LncRNA PVT1 promotes angiogenesis via activating the STAT3/VEGFA axis in gastric cancer. Oncogene 37: 4094-4109, 2018

17. Zhan HX, Wang Y, Li C, Xu JW, Zhou B, Zhu JK, Han HF, Wang L, Wang YS and Hu SY: LincRNA-ROR promotes invasion, metastasis and tumor growth in pancreatic cancer through activating ZEB1 pathway. Cancer Lett 374: 261-271, 2016.

18. Shi X, Wang $X$ and Hua Y: LncRNA GACAT1 promotes gastric cancer cell growth, invasion and migration by regulating MiR-149-mediated of ZBTB2 and SP1. J Cancer 9: 3715-3722, 2018.

19. Wang HM, Lu JH, Chen WY and Gu AQ: Upregulated lncRNA-UCA1 contributes to progression of lung cancer and is closely related to clinical diagnosis as a predictive biomarker in plasma. Int J Clin Exp Med 8: 11824-11830, 2015.

20. Wang YH, Ji J, Wang BC, Chen H, Yang ZH, Wang K, Luo CL, Zhang WW, Wang FB and Zhang XL: Tumor-derived exosomal long noncoding RNAs as promising diagnostic biomarkers for prostate cancer. Cell Physiol Biochem 46: 532-545, 2018.

21. Elsayed ET, Salem PE, Darwish AM and Fayed HM: Plasma long non-coding RNA HOTAIR as a potential biomarker for gastric cancer. Int J Biol Markers: Apr 1, 2018 (Epub ahead of print). doi: $10.1177 / 1724600818760244$

22. Zhu M, Wang Y, Liu X, Wen X, Liang $C$ and Tu J: LncRNAs act as prognostic biomarkers in gastric cancer: A systematic review and meta-analysis. Front Lab Med 1: 59-68, 2017.

23. Iranpour M, Soudyab M, Geranpayeh L, Mirfakhraie R, Azargashb E, Movafagh A and Ghafouri-Fard S: Expression analysis of four long noncoding RNAs in breast cancer. Tumour Biol 37: 2933-2940, 2016.

24. Zhao W, Luo J and Jiao S: Comprehensive characterization of cancer subtype associated long non-coding RNAs and their clinical implications. Sci Rep 4: 6591, 2014.

25. Li J, Lian Y, Yan C, Cai Z, Ding J, Ma Z, Peng P and Wang K: Long non-coding RNA FOXP4-AS1 is an unfavourable prognostic factor and regulates proliferation and apoptosis in colorectal cancer. Cell Prolif 50, 2017.

26. He W, Zhong G, Jiang N, Wang B, Fan X, Chen C, Chen X, Huang $\mathrm{J}$ and Lin T: Long noncoding RNA BLACAT2 promotes bladder cancer-associated lymphangiogenesis and lymphatic metastasis. J Clin Invest 128: 861-875, 2018.

27. Yang ZT, Li Z, Wang XG, Tan T, Yi F, Zhu H, Zhao JP and Zhou XF: Overexpression of long non-coding RNA ZXF2 promotes lung adenocarcinoma progression through c-Myc pathway. Cell Physiol Biochem 35: 2360-2370, 2015.

28. Thai P, Statt S, Chen $\mathrm{CH}$, Liang E, Campbell $\mathrm{C}$ and $\mathrm{Wu} \mathrm{R}$ : Characterization of a novel long noncoding RNA, SCAL1, induced by cigarette smoke and elevated in lung cancer cell lines. Am J Respir Cell Mol Biol 49: 204-211, 2013. 
29. Beckedorff FC, Ayupe AC, Crocci-Souza R, Amaral MS, Nakaya HI, Soltys DT, Menck CF, Reis EM and VerjovskiAlmeida S: The intronic long noncoding RNA ANRASSF1 recruits PRC2 to the RASSF1A promoter, reducing the expression of RASSF1A and increasing cell proliferation. PLoS Genet 9: e1003705, 2013.

30. Livak KJ and Schmittgen TD: Analysis of relative gene expression data using real-time quantitative PCR and the 2(-Delta Delta C(T)) method. Methods 25: 402-408, 2001.

31. Tang Z, Li C, Kang B, Gao G, Li C and Zhang Z: GEPIA: A web server for cancer and normal gene expression profiling and interactive analyses. Nucleic Acids Res 45: W98-W102, 2017.

32. Györffy B, Lanczky A, Eklund AC, Denkert C, Budczies J, Li Q and Szallasi Z: An online survival analysis tool to rapidly assess the effect of 22,277 genes on breast cancer prognosis using microarray data of 1,809 patients. Breast Cancer Res Treat 123: 725-731, 2010

33. Szász AM, Lánczky A, Nagy Á, Förster S, Hark K, Green JE, Boussioutas A, Busuttil R, Szabó A and Győrffy B: Cross-validation of survival associated biomarkers in gastric cancer using transcriptomic data of 1,065 patients. Oncotarget 7: 49322-49333, 2016

34. Győrffy B, Surowiak P, Budczies J and Lánczky A: Online survival analysis software to assess the prognostic value of biomarkers using transcriptomic data in non-small-cell lung cancer. PLoS One 8: e82241, 2013.

35. Gao J, Aksoy BA, Dogrusoz U, Dresdner G, Gross B, Sumer SO Sun Y, Jacobsen A, Sinha R, Larsson E, et al: Integrative analysis of complex cancer genomics and clinical profiles using the cBioPortal. Sci Signal 6: pl1, 2013.

36. Cooper MG (ed): The development and causes of cancer. In: The Cell: A Molecular Approach. 2nd edition. NCBI Bookshelf. Sunderland, MA, 2000

37. Torre LA, Bray F, Siegel RL, Ferlay J, Lortet-Tieulent J and Jemal A: Global cancer statistics, 2012. CA Cancer J Clin 65 87-108, 2015

38. Yao XM, Tang JH, Zhu $\mathrm{H}$ and Jing Y: High expression of LncRNA CASC15 is a risk factor for gastric cancer prognosis and promote the proliferation of gastric cancer. Eur Rev Med Pharmacol Sci 21: 5661-5667, 2017.

39. Uzan VR, Lengert Av, Boldrini É,Penna V, Scapulatempo-Neto C, Scrideli CA, Filho AP, Cavalcante CE, de Oliveira CZ, Lopes LF and Vidal DO: High expression of HULC is associated with poor prognosis in osteosarcoma patients. PLoS One 11: e0156774, 2016.

40. Dorak MT and Karpuzoglu E: Gender differences in cancer susceptibility: An inadequately addressed issue. Front Genet 3: 268, 2012.

41. Deng JY and Liang H: Clinical significance of lymph node metastasis in gastric cancer. World J Gastroenterol 20: 3967-3975, 2014.

42. Cho JK, Hyun SH, Choi N, Kim MJ, Padera TP, Choi JY and Jeong HS: Significance of lymph node metastasis in cancer dissemination of head and neck cancer. Transl Oncol 8: 119-125, 2015 .
43. Cuschieri A, Joypaul V, Fayers P, Cook P, Fielding J and Craven J: Postoperative morbidity and mortality after D1 and D2 resections for gastric cancer: Preliminary results of the MRC randomised controlled surgical trial. Lancet 347: 995-999, 1996.

44. Shitara K, Yatabe Y, Matsuo K, Sugano M, Kondo C, Takahari D, Ura T, Tajika M, Ito S and Muro K: Prognosis of patients with advanced gastric cancer by HER 2 status and trastuzumab treatment. Gastric Cancer 16: 261-267, 2013.

45. Zhou Q, Hou CN, Yang HJ, He Z and Zuo MZ: Distinct expression and prognostic value of members of the epidermal growth factor receptor family in ovarian cancer. Cancer Manag Res 10: 6937-6948, 2018.

46. Gao F, Zhang X, Whang S and Zheng C: Prognostic impact of plasma ORM2 levels in patients with stage II colorectal cancer. Ann Clin Lab Sci 44: 388-393, 2014.

47. Li P, Zhang X, Wang H, Wang L, Liu T, Du L, Yang Y and Wang C: MALAT1 is associated with poor response to oxaliplatin-based chemotherapy in colorectal cancer patients and promotes chemoresistance through EZH2. Mol Cancer Ther 16: 739-751, 2017.

48. Du L, Duan W, Jiang X, Zhao L, Li J, Wang R, Yan S, Xie Y, Yan K, Wang Q, et al: Cell-free lncRNA expression signatures in urine serve as novel non-invasive biomarkers for diagnosis and recurrence prediction of bladder cancer. J Cell Mol Med 22: 2838-2845, 2018

49. Li C, Li W, Zhang Y, Zhang X, Liu T, Zhang Y, Yang Y, Wang L, Pan H, Ji J and Wang C: Increased expression of antisense lncRNA SPINT1-AS1 predicts a poor prognosis in colorectal cancer and is negatively correlated with its sense transcript. Onco Targets Ther 11: 3969-3978, 2018.

50. Terai G, Iwakiri J, Kameda T, Hamada M and Asai K: Comprehensive prediction of lncRNA-RNA interactions in human transcriptome. BMC Genomics 17 (Suppl 1): S12, 2016.

51. Lei F, Zhang $\mathrm{H}$ and Xie $\mathrm{X}$ : Comprehensive analysis of an lncRNA-miRNA-mRNA competing endogenous RNA network in pulpitis. PeerJ 7: e7135, 2019.

52. Tang XJ, Wang W and Hann SS: Interactions among lncRNAs, miRNAs and mRNA in colorectal cancer. Biochimie 163: 58-72, 2019.

53. Thomas LR, Wang Q, Grieb BC, Phan J, Foshage AM, Sun Q Olejniczak ET, Clark T, Dey S, Lorey S, et al: Interaction with WDR5 promotes target gene recognition and tumorigenesis by MYC. Mol Cell 440-452, 2015.

This work is licensed under a Creative Commons Attribution-NonCommercial-NoDerivatives 4.0 International (CC BY-NC-ND 4.0) License. 